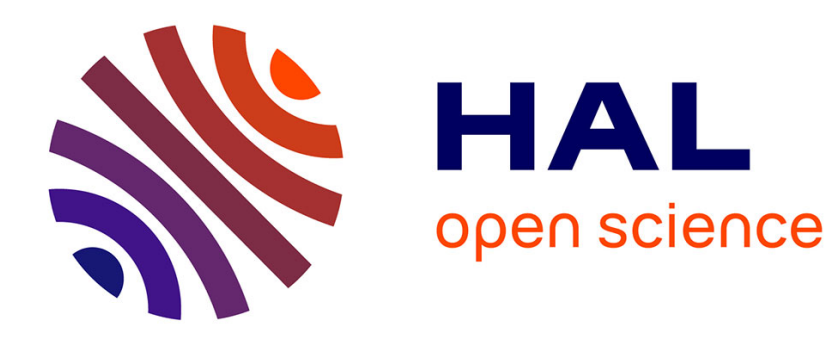

\title{
New applications of the p-adic Nevanlinna Theory
}

Alain Escassut, Ta Thi Hoai An

\section{To cite this version:}

Alain Escassut, Ta Thi Hoai An. New applications of the p-adic Nevanlinna Theory. p-Adic Numbers, Ultrametric Analysis and Applications, 2018. hal-01921858

\section{HAL Id: hal-01921858 \\ https://hal.uca.fr/hal-01921858}

Submitted on 14 Nov 2018

HAL is a multi-disciplinary open access archive for the deposit and dissemination of scientific research documents, whether they are published or not. The documents may come from teaching and research institutions in France or abroad, or from public or private research centers.
L'archive ouverte pluridisciplinaire HAL, est destinée au dépôt et à la diffusion de documents scientifiques de niveau recherche, publiés ou non, émanant des établissements d'enseignement et de recherche français ou étrangers, des laboratoires publics ou privés. 


\title{
New applications of the p -adic Nevanlinna Theory
}

\author{
Alain Escassut and Ta Thi Hoai An
}

\begin{abstract}
Let $\mathbb{K}$ be an algebraically closed field of characteristic 0 complete for an ultrametric absolute value. Following results obtained in complex analysis, here we examine problems of uniqueness for meromorphic functions having finitely many poles, sharing points or a pair of sets (C.M. or I.M.) defined either in the whole field $\mathbb{K}$ or in an open disk, or in the complement of an open disk. Following previous works in $\mathbb{C}$, we consider functions $f^{n}(x) f^{m}(a x+b), g^{n}(x) g^{m}(a x+b)$ with $|a|=1$ and $n \neq m$, sharing a rational function and we show that $\frac{f}{g}$ is a $n+m$-th root of 1 whenever $n+m \geq 5$. Next, given a small function $w$, if $n, m \in \mathbb{N}$ are such that $|n-m|_{\infty} \geq 5$, then $f^{n}(x) f^{m}(a x+b)-w$ has infinitely many zeros. Finally, we examine branched values for meromorphic functions $f^{n}(x) f^{m}(a x+b)$.
\end{abstract}

\section{Introduction}

Let $\mathbb{K}$ be a complete ultrametric algebraically closed field of characteristic 0 whose ultrametric absolute value is denoted by $|$.$| . The Nevanlinna theory,$ well known for complex meromorphic functions [19], was examined over IK by Ha Huy Khoai [9] and A. Boutabaa [3]. Next, in [4] a similar theory was made for unbounded meromorphic functions in an "open" disk of $\mathbb{K}$, taking into account Lazard's problem. In [10], M. O. Hanyak and A. A. Kondratyuk constructed a Nevanlinna theory for meromorphic functions in a set of the form $\mathbb{C} \backslash\left\{a_{1}, \ldots, a_{m}\right\}$, where the meromorphic functions can admit essential singularities at $a_{1}, \ldots, a_{m}$ [10].

Here we recall the Nevanlinna theory for meromorphic functions in the complement of an open disk. Next, we can apply this to obtain results on uniqueness and branched values as it was done in similar problems [1], [2], [3], [3], [4]. We then show new results on meromorphic functions sharing two sets and properties of meromorphic functions of the form $f^{n}(x) f^{m}(a x+b)$ with regards to branched values and Picard's values.

\footnotetext{
${ }^{0} 2010$ Mathematics Subject Classification: 12J25; 30D35; 30G06; 46 S10

${ }^{0}$ Keywords: P-adic meromorphic functions, Nevanlinna's Theory, Values distribution, small functions, Picard values, Branched values.

${ }^{0}$ The second named author is supported by Vietnam's National Foundation for Science and Technology Development (NAFOSTED) under grant number 101.04-2017.320 and Vietnam Institute Advanced Study in Mathematics
} 
All results of Paragraphs 1 to 7 were already published with all proofs in [2], $[4],[7],[8],[14]$. Therefore we will only give the proofs of theorems presented in Paragraph 8.

Notation: Given $r>0, a \in \mathbb{K}$ we denote by $d(a, r)$ the disk $\{x \in \mathbb{K}|| x-a \mid \leq$ $r\}$, by $d\left(a, r^{-}\right)$the disk $\{x \in \mathbb{K}|| x-a \mid<r\}$, and by $C(a, r)$ the circle $\{x \in \mathbb{K}|| x-a \mid=r\}$. Given $r^{\prime \prime}>r^{\prime}$, we put $\Delta\left(0, r^{\prime}, r^{\prime \prime}\right)=d\left(0, r^{\prime \prime}\right) \backslash d\left(0, r^{-}\right)$.

Henceforth, we fix $R>0$, we denote by $S$ the disk $d\left(0, R^{-}\right)$and put $D=$ $\mathbb{K} \backslash S$.

Given a subset $E$ of $\mathbb{K}$ having infinitely many points, we denote by $R(E)$ the $\mathbb{K}$-algebra of rational functions $h \in \mathbb{K}(x)$ having no pole in $E$. We then denote by $H(E)$ the $\mathbb{K}$-vector space of analytic elements on $E$, i.e., the completion of $R(E)$ with respect to the topology of uniform convergence on $E$. By classical properties of analytic elements in [12], [7], given a circle $C(a, r)$ and an element $f$ of $H(C(a, r))$, i.e., a Laurent series $f(x)=\sum_{-\infty}^{+\infty} c_{n}(x-a)^{n}$ converging whenever $|x-a|=r$, then $|f(x)|$ is equal to $\sup _{n \in \mathbb{Z}}\left|c_{n}\right| r^{n}$ in all classes of $C(a, r)$ except maybe in finitely many. When $a=0$, we put $|f|(r)=\sup _{n \in \mathbb{Z}}\left|c_{n}\right| r^{n}$.

We denote by $\mathcal{A}(\mathbb{K})$ the $\mathbb{K}$-algebra of entire functions in $\mathbb{K}$, by $\mathcal{A}\left(d\left(a, R^{-}\right)\right.$ the $\mathbb{K}$-algebra of power series $\sum_{n=0}^{\infty} c_{n}(x-a)^{n}$ converging in all $d\left(a, R^{-}\right)$and by $\mathcal{A}(D)$ the $\mathbb{K}$-algebra of Laurent series $\sum_{-\infty}^{\infty} c_{n}(x-a)^{n}$ converging in $D$. Similarly, we denote by $\mathcal{M}(\mathbb{K})$ the field of meromorphic functions in $\mathbb{K}$, i.e. the field of fractions of $\mathcal{A}(\mathbb{K})$, by $\mathcal{M}\left(d\left(a, R^{-}\right)\right)$the field of meromorphic functions in $d\left(a, R^{-}\right)$i.e. the field of fractions of $\mathcal{A}\left(d\left(a, R^{-}\right)\right)$, and by $\mathcal{M}(D)$ the field of meromorphic functions in $D$ i.e. the field of fractions of $\mathcal{A}(D)$.

Next, we denote by $\mathcal{A}_{b}\left(d\left(a, R^{-}\right)\right)$, the set of $f \in \mathcal{A}\left(d\left(a, R^{-}\right)\right)$that are bounded in $d\left(a, R^{-}\right)$and we put $\mathcal{A}_{u}\left(d\left(a, R^{-}\right)\right)=\mathcal{A}\left(d\left(a, R^{-}\right)\right) \backslash \mathcal{A}_{b}\left(d\left(a, R^{-}\right)\right)$. We denote by $\mathcal{M}_{b}\left(d\left(a, R^{-}\right)\right)$the field of fractions of $\mathcal{A}_{b}\left(d\left(a, R^{-}\right)\right)$and put $\mathcal{M}_{u}\left(d\left(a, R^{-}\right)\right)=\mathcal{M}\left(d\left(a, R^{-}\right)\right) \backslash \mathcal{M}_{b}\left(d\left(a, R^{-}\right)\right)$.

We denote by $\mathcal{A}^{z}(D)$ the set of $f \in \mathcal{A}(D)$ admitting finitely many zeros in $D$ and we put $\mathcal{A}^{*}(D)=\mathcal{A}(D) \backslash \mathcal{A}^{z}(D)$ and similarly, we denote by $\mathcal{M}^{z}(D)$ the field of fraction of $\mathcal{A}^{z}(D)$ and we put $\mathcal{M}^{*}(D)=\mathcal{M}(D) \backslash \mathcal{M}^{z}(D)$. So, $\mathcal{M}^{*}(D)$ is the set of meromorphic functions in $D$ having at least infinitely many zeros or infinitely many poles in $D$.

\section{Nevanlinna Theory in the classical p-adic con- text}

Definition and notation: Let $f \in \mathcal{M}\left(d\left(0, R^{-}\right)\right.$) (resp. $\left.f \in \mathcal{M}(D)\right)$ and let $\alpha \in d\left(a, R^{-}\right)$, resp. $\left.\alpha \in D\right)$. If $f$ admits a zero of order $q$ at $\alpha$ we set $\omega_{\alpha}(f)=q$ and if $f$ has no zero and no pole at $\alpha$, we set $\omega_{\alpha}(f)=0$.

Let $f=\frac{h}{l} \in \mathcal{M}\left(d\left(a, R^{-}\right)\right)$, (resp. $\left.f \in \mathcal{M}(D)\right)$. For each $\alpha \in \mathbb{K}$ (resp. $\alpha \in d\left(a, R^{-}\right)$, resp. $\left.\alpha \in D\right)$ the number $\omega_{\alpha}(h)-\omega_{\alpha}(l)$ does not depend on the 
functions $h, l$ choosed to make $f=\frac{h}{l}$. Thus, we can generalize the notation by setting $\omega_{\alpha}(f)=\omega_{\alpha}(h)-\omega_{\alpha}(l)$.

If $\omega_{\alpha}(f)$ is an integer $q>0, \alpha$ is called a zero of $f$ of order $q$. If $\omega_{\alpha}(f)$ is an integer $q<0, \alpha$ is called a pole of $f$ of order $-q$. If $\omega_{\alpha}(f) \geq 0, f$ is said to be holomorphic at $\alpha$.

Throughout the next paragraphs, we denote by $I$ the interval $[t,+\infty[$, by $J$ an interval of the form $[t, R[$ with $t>0$. We denote by $f$ a function that belongs either to $\mathcal{M}(\mathbb{K})$ or to $\mathcal{M}(S)$.

We have to introduce the counting function of zeros and poles of $f$, counting or not multiplicity. Here we will choose a presentation that avoids assuming that all functions we consider admit no zero and no pole at the origin.

Definitions: We denote by $Z(r, f)$ the counting function of zeros of $f$ in $d(0, r)$ in the following way.

Let $\left(a_{n}\right), 1 \leq n \leq \sigma(r)$ be the finite sequence of zeros of $f$ such that $0<\left|a_{n}\right| \leq r$, of respective order $s_{n}$.

We set $Z(r, f)=\max \left(\omega_{0}(f), 0\right) \log r+\sum_{n=1}^{\sigma(r)} s_{n}\left(\log r-\log \left|a_{n}\right|\right)$ and so, $Z(r, f)$ is called the counting function of zeros of $f$ in $d(0, r)$, counting multiplicity.

In order to define the counting function of zeros of $f$ without multiplicity, we put $\overline{\omega_{0}}(f)=0$ if $\omega_{0}(f) \leq 0$ and $\overline{\omega_{0}}(f)=1$ if $\omega_{0}(f) \geq 1$.

Now, set $\bar{Z}(r, f)=\overline{\omega_{0}}(f) \log r+\sum_{n=1}^{\sigma(r)}\left(\log r-\log \left|a_{n}\right|\right)$ and so, $\bar{Z}(r, f)$ is called the counting function of zeros of $f$ in $d(0, r)$ ignoring multiplicity.

In the same way, considering the finite sequence $\left(b_{n}\right), 1 \leq n \leq \tau(r)$ of poles of $f$ such that $0<\left|b_{n}\right| \leq r$, with respective multiplicity order $t_{n}$, we put

$N(r, f)=\max \left(-\omega_{0}(f), 0\right) \log r+\sum_{n=1}^{\tau(r)} t_{n}\left(\log r-\log \left|b_{n}\right|\right)$ and then $N(r, f)$ is called the counting function of the poles of $f$, counting multiplicity.

Next, we put $\overline{\overline{\omega_{0}}}(f)=0$ if $\omega_{0}(f) \geq 0$ and $\overline{\overline{\omega_{0}}}(f)=1$ if $\omega_{0}(f) \leq-1$ and we set $\bar{N}(r, f)=\overline{\overline{\omega_{0}}}(f) \log r+\sum_{n=1}^{\tau(r)}\left(\log r-\log \left|b_{n}\right|\right)$ and then $\bar{N}(r, f)$ is called the counting function of the poles of $f$, ignoring multiplicity.

Now, we can define the Nevanlinna function $T(r, f)$ in $I$ or $J$ as $T(r, f)=$ $\max (Z(r, f), N(r, f))$ and the function $T(r, f)$ is called characteristic function of $f$ or Nevanlinna function of $f$.

Finally, if $Y$ is a subset of $\mathbb{K}$ we will denote by $Z^{Y}\left(r, f^{\prime}\right)$ the counting function of zeros of $f^{\prime}$, excluding those which are zeros of $f-a$ for any $a \in Y$.

Remark: If we change the origin, the functions $Z, N, T$ are not changed, up to an additive constant.

Theorem 3.1: Let $f \in \mathcal{M}(\mathbb{K})$ (resp. $f \in \mathcal{M}\left(d\left(0, R^{-}\right)\right)$) have no zero and no pole at 0 . Then $\log (|f|(r))=\log (|f(0)|)+Z(r, f)-N(r, f)$. 
Theorem 3.2 (First Main Theorem) : Let $f, g \in \mathcal{M}(\mathbb{K})$ (resp. $f, g \in$ $\mathcal{M}(S))$. Then $Z(r, f g) \leq Z(r, f)+Z(r, g), N(r, f g) \leq N(r, f)+N(r, g)$, and $T(r, f+b)=T(r, f)+O(1), T(r, f g) \leq T(r, f)+T(r, g), T(r, f+g) \leq T(r, f)+$ $\left.T(r, g)+O(1), T(r, c f)=T(r, f) \forall c \in \mathbb{K}^{*}, T\left(r, \frac{1}{f}\right)=T(r, f)\right), T\left(r, \frac{f}{g}\right) \leq$ $T(r, f))+T(r, g)$. Let $P(X) \in \mathbb{K}[X]$. Then $T(r, P(f))=\operatorname{deg}(P) T(r, f)+O(1)$ and $T\left(r, f^{\prime} P(f) \geq T(r, P(f))\right.$.

Suppose now $f, g \in \mathcal{A}(\mathbb{K})$ (resp. $f, g \in \mathcal{A}(S)$ ). Then $Z(r, f g)=Z(r, f)+$ $Z(r, g), T(r, f)=Z(r, f)) T(r, f g)=T(r, f)+T(r, g)+O(1)$ and

$T(r, f+g) \leq \max (T(r, f), T(r, g))$.

Moreover, if $\lim _{r \rightarrow+\infty} T(r, f)-T(r, g)=+\infty$ then $T(r, f+g)=T(r, f)$ when $r$ is big enough.

Theorem 3.3: Let $f \in \mathcal{M}(\mathbb{K})$. Then $f$ belongs to $\mathbb{K}(x)$ if and only if $T(r, f)=O(\log r)$.

Corollary 3.3.a: Let $f \in \mathcal{M}^{*}(\mathbb{K})$. Then $f$ is transcendental over $\mathbb{K}(x)$.

Theorem 3.4: Let $f \in \mathcal{M}(S)$. Then $f$ belongs to $\mathcal{M}_{b}(S)$ if and only if $T(r, f)$ is bounded in $[0, R[$.

Corollary 3.4.a: Let $f \in \mathcal{M}_{u}(S)$. Then $f$ is transcendental over $\mathcal{M}_{b}(S)$.

Theorem 3.5: $\quad$ Let $f \in \mathcal{M}(\mathbb{K})$ (resp. $f \in \mathcal{M}(S)$ ). Then for all $k \in \mathbb{N}^{*}$, we have $N\left(r, f^{(k)}\right)=N(r, f)+k \bar{N}(r, f)$ and $Z\left(r, f^{(k)}\right) \leq Z(r, f)+k \bar{N}(r, f)-$ $k \log (r)+O(1)$.

Theorem 3.6: $\quad$ Let $f \in \mathcal{M}(\mathbb{K})$ (resp. $f \in \mathcal{M}(S)$ ) and let $a_{1}, \ldots, a_{q} \in \mathbb{K}$ be distinct. Then $(q-1) T(r, f) \leq \max _{1 \leq k \leq q}\left(\sum_{j=1, j \neq k}^{q} Z\left(r, f-a_{j}\right)\right)+O(1)$.

Remark: The last Theorem does not hold in complex analysis. Indeed, let $f$ be a meromorphic function in $\mathbb{C}$ omitting two values $a$ and $b$, such as $f(x)=$ $\frac{e^{x}}{e^{x}-1}$. Then $Z(r, f-a)+Z(r, f-b)=0$.

We can now state the Second Main Theorem.

Theorem 3.7: (Second Main Theorem) [3], [4], [7] Let $\alpha_{1}, \ldots, \alpha_{q} \in \mathbb{K}$, with $q \geq 2$, let $Y=\left\{\alpha_{1}, \ldots, \alpha_{q}\right\}$ and let $f \in \mathcal{M}(\mathbb{K})$ (resp. $f \in \mathcal{M}_{u}(S)$ ). Then $(q-1) T(r, f) \leq \sum_{j=1}^{q} \bar{Z}\left(r, f-\alpha_{j}\right)+\bar{N}(r, f)-Z_{0}^{Y}\left(r, f^{\prime}\right)-\log r+O(1) \quad \forall r \in I$ (resp. $\forall r \in J)$.

Moreover, if $f$ belongs to $f \in \mathcal{A}(\mathbb{K})$ (resp. $f \in \mathcal{A}(S)$ ), then $(q-1) T(r, f)$ $\leq \sum_{j=1}^{q} \bar{Z}\left(r, f-\alpha_{j}\right)-Z_{0}^{Y}\left(r, f^{\prime}\right)-\log (r)+O(1) \quad \forall r \in I \quad($ resp. $\forall r \in J)$. 


\section{Nevanlinna Theory out of hole}

Henceforth, we denote by $L$ the interval $[R,+\infty[$. According to classical properties of analytic elements on infraconnected sets and by implicitly using [7], it is easy to have the following properties:

Proposition 4.1: Let $f \in H(D)$ have no zero in $D$. Then $f(x)$ is of the form $\sum_{-\infty}^{q} a_{n} x^{n}$ with $\left|a_{q}\right| R^{q}>\left|a_{n}\right| R^{n}$ for all $n<q$.

Definition and notation: Let $f \in H(D)$ have no zero in $D, f(x)=\sum_{-\infty}^{q} a_{n} x^{n}$ with $\left|a_{q}\right| R^{q}>\left|a_{n}\right| R^{n}$ for all $n<q$ and $a_{q}=1$. Then $f$ will be called a Motzkin factor associated to $S$ and the integer $q$ will be called the Motzkin index of $f$ and will be denoted by $m(f, S)$ (see [2], [7], [14]). We put $L=[R,+\infty[$.

Theorem 4.2: Let $f \in \mathcal{M}(D)$. We can write $f$ in a unique way in the form $f^{S} f^{0}$ with $f^{S} \in H(D)$ a Motzkin factor associated to $S$ and $f^{0} \in \mathcal{M}(\mathbb{K})$, having no zero and no pole in $S$.

Given $f \in \mathcal{M}(D)$, for $r>R$. If $\alpha_{1}, \ldots, \alpha_{m}$ are the distinct zeros of $f$ in $\Delta(0, R, r)$, with respective multiplicity $u_{j}, 1 \leq j \leq m$, then the counting function of zeros $Z_{R}(r, f)$ of $f$ between $R$ and $r$ will be denoted by $Z_{R}(r, f)=\sum_{j=1}^{m} u_{j}\left(\log (r)-\log \left(\left|\alpha_{j}\right|\right)\right)$. Similarly, if $\beta_{1}, \ldots, \beta_{n}$ are the distinct poles of $f$ in $\Delta(0, R, r)$, with respective multiplicity $v_{j}, 1 \leq j \leq m$, then the counting function of poles $N_{R}(r, f)$ of $f$ between $R$ and $r$ will be denoted by $N_{R}(r, f)=\sum_{j=1}^{n} v_{j}\left(\log (r)-\log \left(\left|\beta_{j}\right|\right)\right)$. We put $T_{R}(r, f)=\max \left(Z_{R}(r, f), N_{R}(r, f)\right)$.

The counting function of zeros without counting multiplicity $\bar{Z}_{R}(r, f)$ is defined as: $\bar{Z}_{R}(r, f)=\sum_{j=1}^{m} \log (r)-\log \left(\left|\alpha_{j}\right|\right)$, where $\alpha_{1}, \ldots, \alpha_{m}$ are the distinct zeros of $f$ in $\Delta(0, R, r)$. Similarly, the counting function of poles without counting multiplicity $\bar{N}_{R}(r, f)$ is defined as: $\bar{N}_{R}(r, f)=\sum_{j=1}^{n} \log (r)-\log \left(\left|\beta_{j}\right|\right)$, where $\beta_{1}, \ldots, \beta_{n}$ are the distinct poles of $f$ in $\Delta(0, R, r)$.

Finally, putting $Y=\left\{a_{1}, \ldots, a_{q}\right\}$, we denote by $Z_{R}^{Y}\left(r, f^{\prime}\right)$ the counting function of zeros of $f^{\prime}$ on points $x$ where $f(x) \notin Y$.

Theorem 4.3: Let $f \in \mathcal{M}(D)$. Then, for all $r \in L, \log (|f|(r))-\log (|f|(R))$

$$
=Z_{R}(r, f)-N_{R}(r, f)+m(f, S)(\log r-\log R) .
$$

Corollary 4.3.a Let $f \in \mathcal{M}(D)$. Then $T_{R}(r, f)$ is identically zero if and only if $f$ is a Motzkin factor. Let $f, g \in \mathcal{A}(D)$ satisfy $\log (|f|(r)) \leq \log (|g|(r))$ for all $r \in L$. Then $Z_{R}(r, f) \leq Z_{R}(r, g)+(m(g, S)-m(f, S))(\log (r)-\log (R))$.

Theorem 4.4: Let $f \in \mathcal{A}(D)$. Then, $Z_{R}\left(r, f^{\prime}\right) \leq Z_{R}(r, f)-\log (r)+O(1),(r \in$ $L)$. 
We can now characterize the set $\mathcal{M}^{*}(D)$ :

Theorem 4.5: Let $f \in \mathcal{M}(D)$. The three following statements are equivalent:

i) $\lim _{r \rightarrow+\infty} \frac{T_{R}(r, f)}{\log (r)}=+\infty$ for $r \in L$,

ii) $\frac{T_{R}(r, f)}{\log (r)}$ is unbounded,

iii) $f$ belongs to $\mathcal{M}^{*}(D)$.

Operations on $\mathcal{M}(D)$ work almost like for meromorphic functions in the whole field [3], [7].

Theorem 4.6: If $f, g \in \mathcal{M}(D)$. Then for every $b \in \mathbb{K}$ and $r \in L$, we have $T_{R}\left(r, f^{n}\right)=n T_{R}(r, f), \quad T_{R}(r, f . g) \leq T_{R}(r, f)+T_{R}(r, g)+O(\log (r))$,

$\left.T_{R}\left(r, \frac{1}{f}\right)=T_{R}(r, f)\right), \quad T_{R}(r, f+g) \leq T_{R}(r, f)+T_{R}(r, g)+O(\log (r))$,

$T_{R}(r, f+b)=T_{R}(r, f)+O(\log (r)), T_{R}(r, h \circ f)=T_{R}(r, f)+O(\log (r))$, where $h$ is a Moebius function.

Moreover, if both $f$ and $g$ belong to $\mathcal{A}(D)$, then

$T_{R}(r, f+g) \leq \max \left(T_{R}(r, f), T_{R}(r, g)\right)+O(\log (r)), \quad T_{R}(r, f g)=T_{R}(r, f)+$ $T_{R}(r, g)$. Particularly, if $f \in \mathcal{A}^{*}(D)$, then $T_{R}(r, f+b)=T_{R}(r, f)+O(1)$.

Given a polynomial $P(x) \in \mathbb{K}[x]$, then $T_{R}(r, P \circ f)=\operatorname{deg}(P) T_{R}(r, f)+$ $O(\log (r))$.

Theorem 4.7: Every $f \in \mathcal{M}^{*}(D)$ is transcendental over $\mathcal{M}^{z}(D)$.

Theorem 4.8: Let $f \in \mathcal{M}(D)$. Then, for $r \in L, N_{R}\left(r, f^{(k)}\right)=N_{R}(r, f)+$ $k \bar{N}_{R}(r, f)$ and $Z_{R}\left(r, f^{(k)}\right) \leq Z_{R}(r, f)+k \bar{N}_{R}(r, f)+O(\log (r))$.

Like in the whole field, the Nevanlinna second Main Theorem is based on the following theorem:

Theorem 4.9: Let $f \in \mathcal{M}(D)$ and let $a_{1}, \ldots, a_{q} \in \mathbb{K}$ be distinct. Then $(q-1) T_{R}(r, f) \leq \max _{1 \leq k \leq q}\left(\sum_{j=1, j \neq k}^{q} Z_{R}\left(r, f-a_{j}\right)\right)+O(\log (r))$.

Theorem 4.10: (Second Main Theorem) Let $f \in \mathcal{M}(D)$, let $\alpha_{1}, \ldots, \alpha_{q} \in$ $\mathbb{K}$, with $q \geq 2$ and let $Y=\left\{\alpha_{1}, \ldots, \alpha_{q}\right\}$. Then, for $r \in L,(q-1) T_{R}(r, f)$

$$
\leq \sum_{j=1}^{q} \bar{Z}_{R}\left(r, f-\alpha_{j}\right)+\bar{N}_{R}(r, f)-Z_{R}^{Y}\left(r, f^{\prime}\right)+O(\log (r)) .
$$

Particularly, if $f \in \mathcal{A}(D)$, then

$$
(q-1) T_{R}(r, f) \leq \sum_{j=1}^{q} \bar{Z}_{R}\left(r, f-\alpha_{j}\right)-Z_{R}^{Y}\left(r, f^{\prime}\right)+O(\log (r)) .
$$

\section{Immediate applications}

Theorem 5.1: $\quad$ Let $a_{1}, a_{2} \in \mathbb{K}\left(\right.$ with $\left.a_{1} \neq a_{2}\right)$ and let $f, g \in \mathcal{A}^{*}(\mathbb{K})$ satisfy $f^{-1}\left(\left\{a_{i}\right\}\right)=g^{-1}\left(\left\{a_{i}\right\}\right)(i=1,2)$. Then $f=g$. 
Theorem 5.2: $\quad$ Let $a_{1}, a_{2}, a_{3} \in \mathbb{K}$ (with $a_{i} \neq a_{j} \forall i \neq j$ ) and let $f, g \in$ $\mathcal{A}_{u}\left(d\left(0, R^{-}\right)\right)$(resp. $\left.f, g \in \mathcal{A}^{*}(D)\right)$ satisfy $f^{-1}\left(\left\{a_{i}\right\}\right)=g^{-1}\left(\left\{a_{i}\right\}\right)(i=1,2,3)$. Then $f=g$.

Theorem 5.3: Let $a_{1}, a_{2}, a_{3}, a_{4} \in \mathbb{K}$ (with $a_{i} \neq a_{j} \forall i \neq j$ ) and let $f, g \in$ $\mathcal{M}^{*}(\mathbb{K})$ satisfy $f^{-1}\left(\left\{a_{i}\right\}\right)=g^{-1}\left(\left\{a_{i}\right\}\right)(i=1,2,3,4)$. Then $f=g$.

Theorem 5.4: Let $a_{1}, a_{2}, a_{3}, a_{4}, a_{5} \in \mathbb{K}$ (with $a_{i} \neq a_{j} \forall i \neq j$ ) and let $f, g \in \mathcal{M}_{u}\left(d\left(0, R^{-}\right)\right)$(resp. $\left.f, g \in \mathcal{M}^{*}(D)\right)$ satisfy $f^{-1}\left(\left\{a_{i}\right\}\right)=g^{-1}\left(\left\{a_{i}\right\}\right)$ $(i=1,2,3,4,5)$. Then $f=g$.

Theorem 5.5: Let $\Lambda$ be a non-degenerate elliptic curve of equation $y^{2}=\left(x-a_{1}\right)\left(x-a_{2}\right)\left(x-a_{3}\right)$.

There do not exist $g, f \in \mathcal{M}(\mathbb{K})$ such that $g(t)=y, f(t)=x, t \in \mathbb{K}$.

There do not exist $g, f \in \mathcal{A}_{u}\left(d\left(0, R^{-}\right)\right)$such that $g(t)=y, f(t)=x, t \in$ $d\left(0, R^{-}\right)$.

There do not exist $g, f \in \mathcal{A}^{*}(D)$ such that $g(t)=y, f(t)=x, t \in D$.

Theorem 5.6: Let $\Lambda$ be a curve of equation $y^{q}=P(x), q \geq 2$, with $P \in \mathbb{K}[x]$ admitting $n$ distinct zeros of order 1 with $n \geq 4$. There do not exist $g, f \in$ $\mathcal{M}(\mathbb{I K})$ such that $g(t)=y, f(t)=x, t \in \mathbb{K}$.

Theorem 5.7: Let $\Lambda$ be a curve of equation $y^{q}=P(x), q \geq 2$, with $P \in \mathbb{K}[x]$ admitting $n$ distinct zeros of order 1 with $n \geq 5$. There do not exist $g, f \in$ $\mathcal{M}_{u}\left(d\left(0, R^{-}\right)\right)$(resp. $\left.g, f \in \mathcal{M}^{*}(D)\right)$ such that $g(t)=y, f(t)=x, t \in d\left(0, R^{-}\right)$ (resp. $t \in D)$.

Another application concerns analytic functions:

Theorem 5.8: Let $f, g \in \mathcal{M}\left(d\left(0, R^{-}\right)\right.$) (resp. $\left.f, g \in \mathcal{M}(D)\right)$ satisfy $g^{m}+$ $f^{n}=1$, with $\min (m, n) \geq 3$ and $\left.\max (m, n) \geq 4\right)$. Then $f$ and $g$ belong to $\mathcal{M}_{b}\left(d\left(0, R^{-}\right)\right.$) (resp. to $\left.\mathcal{M}^{z}(D)\right)$. Moreover, if $f, g \in \mathcal{A}\left(d\left(0, R^{-}\right)\right.$(resp. if $f, g \in \mathcal{A}(D))$ satisfy $g^{m}+f^{n}=1$, with $\min (m, n) \geq 2$ and $(m, n) \neq(2,2)$, then $f$ and $g$ belong to $\mathcal{A}_{b}\left(d\left(0, R^{-}\right)\right.$, (resp to $\left.\mathcal{A}^{z}(D)\right)$.

\section{$5 \quad$ Small functions}

Definitions: For each $f \in \mathcal{M}(\mathbb{K})$, (resp. $f \in \mathcal{M}(S)$, resp. $f \in \mathcal{M}(D)$ ), we will denote by $\mathcal{M}_{f}(\mathbb{K})$ (resp. $\left.\mathcal{M}_{f}(S), \mathcal{M}_{f}(D)\right)$ the set of functions $h \in \mathcal{M}(\mathbb{K})$ (resp. $h \in \mathcal{M}(S), h \in \mathcal{M}(D))$ such that $T(r, h)=o(T(r, f)), r \in I$ (resp. $T(r, h)=o(T(r, f)), r \in J$, resp. $\left.T_{R}(r, h)=o\left(T_{R}(r, f)\right) r \in L\right)$. Similarly, if $f \in \mathcal{A}(\mathbb{K})$ (resp. $f \in \mathcal{A}(S)$, resp. $f \in \mathcal{A}(D)$ ) we will denote by $\mathcal{A}_{f}(\mathbb{K})$ (resp. $\mathcal{A}_{f}(S)$, resp. $\left.\mathcal{A}_{f}(D)\right)$ the set $\mathcal{M}_{f}(\mathbb{K}) \cap \mathcal{A}(\mathbb{K}),\left(\operatorname{resp} . \quad \mathcal{M}_{f}(S) \cap \mathcal{A}(S)\right.$, resp. $\left.\mathcal{M}_{f}(D) \cap \mathcal{A}(D)\right)$.

The elements of $\mathcal{M}_{f}(\mathbb{K})$ (resp. $\mathcal{M}_{f}(S)$, resp. $\left.\mathcal{M}_{f}(D)\right)$ are called small meromorphic functions with respect to $f$. Similarly, if $f \in \mathcal{A}(\mathbb{K})$ (resp. $f \in$ $\mathcal{A}(S)$, resp. $f \in \mathcal{A}(D))$ these functions are called small analytic functions with respect to $f$.

A small function $w$ with respect to a function $f \in \mathcal{M}(\mathbb{K})$ (resp. $f \in \mathcal{M}(S)$, resp. $f \in \mathcal{M}(D)$ ) will be called a quasi-exceptional small function for $f$ if $f-w$ has finitely many zeros in $D$. 
Theorem 7.1: Let $f \in \mathcal{M}^{*}(\mathbb{K})$ (resp. $f \in \mathcal{M}_{u}(S)$, resp. $f \in \mathcal{M}^{*}(D)$ ). Then $f$ admits at most one quasi-exceptional small function. Moreover, if $f$ has finitely many poles, then $f$ admits no quasi-exceptional small function.

Corollary 7.1.a: Let $f \in \mathcal{A}^{*}(\mathbb{K})$ (resp. $f \in \mathcal{A}_{u}(S)$, resp. $f \in \mathcal{A}^{*}(D)$ ). Then $f$ has no quasi-exceptional small function.

The Second Main Theorem for Three Small Functions holds as well as in complex analysis. Notice that this theorem was generalized to any finite set of small functions by K. Yamanoi in complex analysis [16], through methods that have no equivalent on a p-adic field.

Theorem 7.2: Let $f \in \mathcal{M}^{*}(\mathbb{K})$, (resp. $f \in \mathcal{M}_{u}(S)$, resp. $f \in \mathcal{M}^{*}(D)$ and let $w_{1}, w_{2}, w_{3} \in \mathcal{M}_{f}(\mathbb{K})$ (resp. $\in \mathcal{M}_{f}\left(d\left(0, R^{-}\right)\right.$), resp. $\left.\in \mathcal{M}_{f}(D)\right)$ be pairwise distinct. Then: $T(r, f) \leq \sum_{j=1}^{3} \bar{Z}\left(r, f-w_{j}\right)+o(T(r, f))$

(resp. $T(r, f) \leq \sum_{j=1}^{3} \bar{Z}\left(r, f-w_{j}\right)+o(T(r, f))$, resp. $T_{R}(r, f) \leq \sum_{j=1}^{3} \bar{Z}_{R}(r, f-$ $\left.\left.w_{j}\right)+o\left(T_{R}(r, f)\right)\right)$.

Corollary 7.2.a: Let $f \in \mathcal{M}^{*}(\mathbb{K})$ (resp. $f \in \mathcal{M}_{u}(S)$ resp. $f \in \mathcal{M}^{*}(D)$ ) and let $w_{1}, w_{2} \in \mathcal{A}_{f}(\mathbb{K})$ (resp. $w_{1}, w_{2} \in \mathcal{A}_{f}(S)$ resp. $w_{1}, w_{2} \in \mathcal{A}_{f}(D)$ ) be distinct. Then $T(r, f) \leq \bar{Z}\left(r, f-w_{1}\right)+\bar{Z}\left(r, f-w_{2}\right)+\bar{N}(r, f)+o(T(r, f))$, (resp. $T(r, f) \leq \bar{Z}\left(r, f-w_{1}\right)+\bar{Z}\left(r, f-w_{2}\right)+\bar{N}(r, f)+o\left(T_{R}(r, f)\right)$, resp. $\left.T_{R}(r, f) \leq \bar{Z}_{R}\left(r, f-w_{1}\right)+\bar{Z}_{R}\left(r, f-w_{2}\right)+\bar{N}_{R}(r, f)+o\left(T_{R}(r, f)\right)\right)$.

Corollary 7.2.b: Let $f \in \mathcal{A}^{*}(\mathbb{I K})$ (resp. $f \in \mathcal{A}_{u}(S)$, rtesp. $f \in \mathcal{A}^{*}(D)$ ) and let $w_{1}, w_{2} \in \mathcal{A}_{f}(S)$, resp. $\left.w_{1}, w_{2} \in \mathcal{A}_{f}(D)\right)$ be distinct. Then $T(r, f) \leq \bar{Z}\left(r, f-w_{1}\right)+\bar{Z}\left(r, f-w_{2}\right)+o(T(r, f))$, (resp. $T(r, f) \leq \bar{Z}\left(r, f-w_{1}\right)+\bar{Z}\left(r, f-w_{2}\right)+o(T(r, f))$, resp. $\left.T_{R}(r, f) \leq \bar{Z}_{R}\left(r, f-w_{1}\right)+\bar{Z}_{R}\left(r, f-w_{2}\right)+o\left(T_{R}(r, f)\right)\right)$.

Definitions: Let $f \in \mathcal{M}^{*}(\mathbb{K})$ (resp. $f \in \mathcal{M}_{u}\left(d\left(0, R^{-}\right)\right)$, resp. $f \in \mathcal{M}^{*}(D)$ ) and let $w \in \mathcal{M}_{f}(\mathbb{K})$ (resp. $w \in \mathcal{M}_{f}\left(d\left(0, R^{-}\right)\right)$, resp. $\left.w \in \mathcal{M}_{f}(D)\right)$. Then $w$ is called a perfectly branched function with respect to $f$ if all zeros of $f-w$ are multiple except maybe finitely many [7] and $w$ is called a totally branched function with respect to $f$ if all zeros of $f-w$ are multiple, without exception [5]. Particularly, the definition applies to constants. The following Theorem 7.3, 7.4, 7.5 are proved in [7] concerning the sets $f \in \mathcal{M}^{*}(\mathbb{K})$ (resp. $f \in \mathcal{M}_{u}\left(d\left(0, R^{-}\right)\right.$), $f \in \mathcal{A}^{*}(\mathbb{K})$ (resp. $f \in \mathcal{A}_{u}\left(d\left(0, R^{-}\right)\right.$). The proofs concerning $\mathcal{M}^{*}(D)$ and $\mathcal{A}^{*}(D)$ are similar to those concerning $\mathcal{M}^{*}(\mathbb{K})$ and $\mathcal{A}^{*}(\mathbb{K})$ respectively.

Theorem 7.3: Let $f \in \mathcal{M}^{*}(\mathbb{I K})$ (resp. $f \in \mathcal{M}_{u}\left(d\left(0, R^{-}\right)\right.$), resp. $\left.f \in \mathcal{M}^{*}(D)\right)$. Then $f$ admits at most four perfectly branched values.

Theorem 7.4: Let $f \in \mathcal{M}^{*}(\mathbb{K})$ (resp. $f \in \mathcal{M}^{*}(D)$ ) having finitely many poles. Then $f$ admits at most one perfectly branched rational function.

Corollary 7.4.a: Let $f \in \mathcal{A}^{*}(\mathbb{I K})$ (resp. $f \in \mathcal{A}^{*}(D)$ ). Then $f$ admits at most one perfectly branched rational function.

Theorem 7.5: Let $f \in \mathcal{M}_{u}\left(d\left(0, R^{-}\right)\right)$, having finitely many poles. Then $f$ admits at most two perfectly branched rational functions.

Corollary 7.5.a: Let $f \in \mathcal{A}_{u}\left(d\left(0, R^{-}\right)\right)$. Then $f$ admits at most two perfectly branched rational functions. 


\section{New applications of the Nevanlinna Theory}

Definitions: Recall that two functions $f$ and $g$ meromorphic in a set $B$ are said to share a set $X \subset \mathbb{K}$, counting multiplicity, or C.M. in brief, if for each $b \in X$, when $f(x)-b$ has a zero of order $q$ at a point $a \in B$, then there exists $c \in X$ such that $g(x)-c$ also has a zero of order $q$ at $a$. And the functions $f$ and $g$ are said to share $X$ ignoring multiplicity or I.M. in brief, if $f^{-1}(X)=g^{-1}(X)$.

In the same way, two functions $f$ and $g$ meromorphic in a set $B$ are said to share a nonidenticaly zero function $h$ from $B$ to $\mathbb{K}$, counting multiplicity, or C.M. in brief, if $\frac{f}{h}$ and $\frac{g}{h}$ share 1 C.M., i.e. if $f-h$ and $g-h$ have the same zeros with the same multiplicity.

By Theorem 5.3, two meromorphic functions in $\mathbb{K}$ sharing 4 points I.M. are identical, by Theorem 5.4 two meromorphic functions in $S$ or in $D$ sharing 5 points I.M. are identical, by Theorem 5.1, two entire functions sharing 2 points I.M. are identical and by Theorem 5.2 two meromorphic functions in $S$ or in $D$ sharing 3 points I.M. are identical. Here we will first examine two meromorphic functions sharing a few points C.M.

Theorem 8.1: Let $f, g \in \mathcal{M}_{u}(S)$ (resp. let $f, g \in \mathcal{M}^{*}(D)$ ) share C.M. 4 points $a_{j} \in \mathbb{K} \cup\{\infty\}, j=1,2,3,4$. Then $f \equiv g$.

Theorem 8.2: Let $f, g \in \mathcal{M}_{u}(S)$ (resp. let $f, g \in \mathcal{M}^{*}(D)$ ) have finitely many poles and share C.M. 3 points $a_{j} \in \mathbb{K} \cup\{\infty\}, j=1,2,3$. Then $f \equiv g$.

Corollary 8.2.a: Let $f, g \in \mathcal{A}_{u}(S)$ (resp. $f, g \in \mathcal{A}^{*}(D)$ ) share C.M. 3 points $a_{j} \in \mathbb{K}, \quad j=1,2,3$. Then $f \equiv g$.

Theorem 8.3 is not immediate and has a similar version in complex analysis for meromorphic functions provided with a finite growth order that is not integral. Here, we don't need any hypothesis on the growth order.

Theorem 8.3: Let $f, g \in \mathcal{M}^{*}(\mathbb{K})$ share C.M. 3 points $a_{j} \in \mathbb{K} \cup\{\infty\}, j=$ $1,2,3$. Then $f \equiv g$.

In the particular case of functions $f, g \in \mathcal{M}_{u}(S)$ or functions $\left.f, g \in \mathcal{M}^{*}(D)\right)$ having finitely many poles and sharing poles C.M., we can add this theorem:

Theorem 8.4: Let $f, g \in \mathcal{M}_{u}(S)$, (resp. let $f, g \in \mathcal{M}^{*}(D)$ ) have finitely many poles in $S$ (resp. in D) and share C.M. two values $a, b$ and poles. Then $f \equiv g$.

Our main theorems are Theorems 8.5 and 8.6 that follow the same kind of reasoning as in [6]. We denote by $Y_{1}=\left\{\alpha_{1}, \ldots, \alpha_{k}\right\}$ and $Y_{2}=\left\{\beta_{1}, \beta_{2}\right\}$ the two sets satisfying

$$
\text { (H) }\left(\prod_{j=1}^{k}\left(\beta_{1}-\alpha_{j}\right)\right)^{2} \neq\left(\prod_{j=1}^{k}\left(\beta_{2}-\alpha_{j}\right)\right)^{2} \text {. }
$$

Theorem 8.5: Let $f, g \in \mathcal{M}^{*}(\mathbb{K}) \quad$ (resp. let $f, g \in \mathcal{M}_{u}(S)$, resp. let $\left.f, g \in \mathcal{M}^{*}(D)\right)$ have finitely many poles in $\mathbb{K}$ (resp. in $S$, resp. in $D$ ) and share $Y_{1}$ C.M. and $Y_{2}$ I.M. Then $f \equiv g$. 
Corollary 8.5.a: Let $f, g \in \mathcal{M}^{*}(\mathbb{K})$ (resp. let $f, g \in \mathcal{M}_{u}(S)$, resp. let $\left.f, g \in \mathcal{M}^{*}(D)\right)$ have finitely many poles in $\mathbb{K}$ (resp. in $S$, resp in $D$ ) and share a value $\alpha$ C.M. and $Y_{2}$ I.M. If $\left(\alpha-\beta_{1}\right)^{2} \neq\left(\alpha-\beta_{2}\right)^{2}$, then $f \equiv g$.

Corollary 8.5.b: Let $f, g \in \mathcal{A}^{*}(\mathbb{K})$ (resp. let $f, g \in \mathcal{A}_{u}(S)$, resp. let $\left.f, g \in \mathcal{A}^{*}(D)\right)$ and share $Y_{1}$ C.M. and $Y_{2}$ I.M. Then $f \equiv g$.

Corollary 8.5.c: Let $f, g \in \mathcal{A}^{*}(\mathbb{K})$ (resp. let $f, g \in \mathcal{A}_{u}(S)$, resp. let $\left.f, g \in \mathcal{A}^{*}(D)\right)$ and share a value $\alpha$ C.M. and $Y_{2}$ I.M. If $\left(\alpha-\beta_{1}\right)^{2} \neq\left(\alpha-\beta_{2}\right)^{2}$, then $f \equiv g$.

Theorem 8.6: Let $f, g \in \mathcal{M}^{*}(\mathbb{K})$ (resp. let $f, g \in \mathcal{M}_{u}(S)$, resp. let $\left.f, g \in \mathcal{M}^{*}(D)\right)$ have finitely many poles in $\mathbb{K}$ (resp. in $S$, resp. in D) and share $Y_{1}$ I.M. and $Y_{2}$ C.M. Then $f \equiv g$.

Corollary 8.6.a: Let $f, g \in \mathcal{M}^{*}(\mathbb{K})$ (resp. let $f, g \in \mathcal{M}_{u}(S)$, resp. let $\left.f, g \in \mathcal{M}^{*}(D)\right)$ have finitely many poles in $\mathbb{K}$ (resp. in $S$, resp. in D) and share a value $\alpha$ I.M. and $Y_{2}$ C.M. If $\left(\alpha-\beta_{1}\right)^{2} \neq\left(\alpha-\beta_{2}\right)^{2}$, then $f \equiv g$.

Corollary 8.6.b: Let $f, g \in \mathcal{A}^{*}(\mathbb{K})$ (resp. let $f, g \in \mathcal{A}_{u}(S)$, resp. let $\left.f, g \in \mathcal{A}^{*}(D)\right)$ and share $Y_{1}$ I.M. and $Y_{2}$ C.M. Then $f \equiv g$.

Corollary 8.6.c: Let $f, g \in \mathcal{A}^{*}(\mathbb{K})$ (resp. let $f, g \in \mathcal{A}_{u}(S)$, resp. let $\left.f, g \in \mathcal{A}^{*}(D)\right)$ and share a value $\alpha$ I.M. and $Y_{2}$ C.M. If $\left(\alpha-\beta_{1}\right)^{2} \neq\left(\alpha-\beta_{2}\right)^{2}$, then $f \equiv g$.

It is known that if two functions $f, g \in \mathcal{A}(\mathbb{K})$ share separately two values $a, b \in \mathbb{K}$ C.M., then $f \equiv g[7]$ (Theorem 41.1). However, here the hypothesis $f, g$ share $Y_{1}$ and share $Y_{2}$ cannot be compared: for example, concerning $Y_{2}, f$ and $g$ are not supposed to share $\beta_{1}$ or $\beta_{2}$ separately. The same remark applies to meromorphic functions having finitely many poles.

Results of [11] and [13] showed the interest of complex functions of the form $f(x) f(x+b)$. Similar studies were made in [11] and [13] in a $p$-adic field. Here we will generalize that kind of study on the field $\mathbb{K}$. In [13], it is proven that if two complex entire functions $f$ and $g$ are such that $f(x)^{n} f(x+c)$ and $g(x)^{n} g(x+c)$ share 1 C.M. with $n \geq 6$, then either $f g$ is a constant $t_{1}$ such that $t_{1}^{n+1}=1$, or $\frac{f}{g}$ is a constant $t_{2}$ such that $t_{2}^{n+1}=1$. Here, on the field $\mathbb{K}$, we can obtain better results.

On the other hand, in [11], we can find similar results of uniqueness for meromorphic functions on a $p$-adic field involving derivatives, sharing 1 C.M. or I.M., also involving derivatives. Here we will examine functions of the form $f(x)^{n}(f(x+c))^{m}, g(x)^{n}(g(x+c))^{m}$ sharing a rational function and we will look for branched values and quasi-exceptional values of such functions.

Notation: We denote by $\mathbb{N}^{*}$ the set of strictly positive integers. On $\mathbb{Z}$, we denote by $|\cdot|_{\infty}$ the Archimedean absolute value.

Theorem 8.7: Let $a \in C(0,1)$, let $b \in \mathbb{K}$ and let $f, g \in \mathcal{M}^{*}(\mathbb{K})$ have finitely many poles and take $m, n \mathbb{N}^{*}$ with $m \neq n$. If $f^{n}(x) f^{m}(a x+b)$ and $g^{n}(x) g^{m}(a x+$ b) share C.M. a rational function $Q \in \mathbb{K}(x), Q \neq 0$ and if $n+m \geq 5$, then $\frac{f}{g}$ is 
a constant $t$ such that $t^{n+m}=1$. Moreover, if $f, g \in \mathcal{A}^{*}(\mathbb{K})$, if $f^{n}(x) f^{m}(a x+b)$ and $g^{n}(x) g^{m}(a x+b)$ share C.M. a constant $l \neq 0$ and if $n+m \geq 4$, then $\frac{f}{g}$ is a constant $t$ such that $t^{n+m}=1$.

Theorem 8.8: Let $a \in C(0,1)$ and let $b \in S$ (resp. let $b \in \mathbb{K}$ ) and let $f, g \in \mathcal{M}_{u}(S)$ (resp. let $f, g \in \mathcal{M}^{*}(D)$ ) have finitely many poles in $S$ (resp. in $D)$ and take $n, m \in \mathbb{N}^{*}$ with $n \neq m$. If $f^{n}(x) f^{m}(a x+b)$ and $g^{n}(x) g^{m}(a x+b)$ share C.M. a function $\theta \in \mathcal{M}(S)$ (resp. a function $\theta \in \mathcal{M}(D)$ ) having finitely many zeros and poles in $S$ (resp. in D), if $n \geq 5$, then $\frac{f}{g}$ is a constant $t$ such that $t^{n+m}=1$.

In [15] it was shown that given a complex entire function $f$ and $b \in \mathbb{C} \backslash\{0\}$, a function of the form $f^{n}(x) f(x+b)-c$ (with $c \neq 0$ ) has infinitely many zeros in $\mathbb{C}$ provided $n \geq 3$. On the field $\mathbb{K}$, such a result is trivial since an entire functions and even a meromorphic function with finitely many poles (which is not a rational function) takes every value infinitely many times. But we can ask the question regarding in general functions $f \in \mathcal{M}^{*}(\mathbb{K}), f \in \mathcal{M}_{u}(S)$, $f \in \mathcal{M}^{*}(D)$.

Theorem 8.9: Let $f \in \mathcal{M}^{*}(\mathbb{K})$ (resp. $f \in \mathcal{M}_{u}(S)$, resp. $f \in \mathcal{M}^{*}(D)$ ), let $a \in C(0,1)$, let $b \in \mathbb{K}$ (resp. $b \in S$, resp. $b \in \mathbb{K}$ ) and let $w \in \mathcal{M}(\mathbb{K})$ (resp. $w \in \mathcal{M}(S)$, resp. $w \in \mathcal{M}(D))$ be a non identically zero small function with respect to $f$. If $n, m \in \mathbb{N}$ are such that $|n-m|_{\infty} \geq 5$, then $f^{n}(x) f^{m}(a x+b)-w$ has infinitely many zeros in $\mathbb{K}$ (resp. in $S$, resp. in D).

Corollary 8.9.a: Let $f \in \mathcal{M}^{*}(\mathbb{K})$ (resp. $f \in \mathcal{M}_{u}(S)$, resp. $f \in \mathcal{M}^{*}(D)$ ), let $a \in C(0,1)$, let $b \in \mathbb{K}$ (resp. $b \in S$, resp. $b \in \mathbb{K}$ ). If $n, m \in \mathbb{N}$ are such that $|n-m|_{\infty} \geq 5$, then $f^{n}(x) f^{m}(a x+b)$ takes every nonzero value infinitely many times in $\mathbb{K}$ (resp. in $S$, resp. in D).

Remarks: 1) Of course, the hypothesis $w \neq 0$ must not be excluded. Indeed, let $h \in \mathcal{A}^{*}(\mathbb{K})$ and let $f(x)=\frac{1}{h(x)}$. Then a function of the form $f^{n}(x) f(a x+b)$ has no zero in $\mathbb{K}$.

2) On the other hand, it is known and easily seen that if $a=1$ and $b=0$, a function $f^{n}-w$ has infinitely many zeros for every $n \geq 3$ and that $f^{2}$ takes every nonzero value $c$ infinitely many times because given a square root $l$ of $c$, then $f^{2}-c=(f-l)(f+l)$ and at least one of the two values $l$ and $-l$ is taken infinitely many times. We can ask whether a meromorphic function of the form $f^{2}(x)-w$ always has infinitely many zeros when $w$ is not a constant.

3) Concerning Theorem 8.9, it is easily proven that if $f$ is a meromorphic function with finitely many poles and $w$ a small function, then $f-w$ has infinitely many zeros. So it is useless here to add a corollary concerning $f^{n}(x) f^{m}(a x+$ $b)-w$ when $f$ has finitely many poles.

Theorem 8.10: Let $f \in \mathcal{M}^{*}(\mathbb{K})$ (resp. $f \in \mathcal{M}_{u}(S)$, resp. $f \in \mathcal{M}^{*}(D)$ ), let $a \in C(0,1)$, let $b \in \mathbb{K}$ (resp. $b \in S$, resp. $b \in \mathbb{K})$ and let $n, m \in$ $\mathbb{N}^{*}$. If $3|n-m|_{\infty}>2(n+m)+4$, then $f^{n}(x) f^{m}(a x+b)$ does not admit 4 distinct perfectly branched values. Moreover, if $4|n-m|_{\infty}>3(n+m)+4$, then $f^{n}(x) f^{m}(a x+b)$ does not admit 3 distinct perfectly branched values. 
Further, if $f$ belongs to $\mathcal{M}^{*}(\mathbb{K})$ and if $3|n-m|_{\infty} \geq 2(n+m+1)$, then $f^{n}(x) f^{m}(a x+b)$ does not admit 4 distinct totally branched values; moreover, if $4|n-m|_{\infty} \geq 3(n+m)+4$, then $f^{n}(x) f^{m}(a x+b)$ does not admit 3 distinct totally branched values.

\section{$7 \quad$ Tools and Lemmas}

In the proofs of our main theorems, we will use the following lemmas:

Lemma 1: Let $P(x) \mathbb{K}[x]$ have all its zeros of order 1. Let $f \in \mathcal{M}^{*}(\mathbb{K})$ (resp. let $f \in \mathcal{M}_{u}(S)$, resp. let $\left.f \in \mathcal{M}^{*}(D)\right)$ and suppose that $f-b$ admits a zero of order $q$ at a point $u \in \mathbb{K}$ (resp. at a point $u \in S$, resp. at a point $u \in D)$. Then $P(f)-P(b)$ admits $u$ as a zero of order $q$.

Lemmas 2, 3 and 4 are well known [7], Lemma 32.3, Corollary 32.6.

Lemma 2: Let $f \in \mathcal{M}^{*}(\mathbb{I K})$ have finitely many poles. Then for every $Q \in$ $\mathbb{K}(x), f-Q$ has inifinitely many zeros.

Lemma 3: Let $f \in \mathcal{M}^{*}(\mathbb{K})$. There exist $\phi$ and $\psi \in \mathcal{A}(\mathbb{K})$ having no common zero, such that $f=\frac{\phi}{\psi}$. Moreover, if $f$ has no zero and no pole then $f$ is a constant.

Lemma 4 is a consequence of Theorem 40.10 and Theorem 43.1 in [7]:

Lemma 4: Let $w \in \mathcal{M}(S)$ have finitely many zeros and poles in $S$. Then $w$ belongs to $\mathcal{M}_{b}(S)$. Moreover, given $f \in \mathcal{M}_{u}(S)$ having finitely many poles in $S$, then $f-w$ belongs to $\mathcal{M}_{u}(S)$ and has infinitely many zeros.

Lemma 5: Let $f(x) \in \mathcal{A}(D)$. Then $f$ belongs to $\mathcal{A}^{z}(D)$ if and only if it is of the form $\sum_{-\infty}^{q} a_{n} x^{n}$ with $q \in \mathbb{Z}$ and $\lim _{n \rightarrow-\infty}\left|a_{n}\right| R^{n}=0$. Moreover, given $f \in \mathcal{M}^{*}(D)$ having finitely many poles in $D$ and $w \in \mathcal{M}^{z}(D)$, then $f-w$ has infinitely many zeros.

Lemma 6 is immediate :

Lemma 6: Let $u \in C(0,1)$ and $c \in \mathbb{K}$ (resp. $c \in S$, resp. $c \in \mathbb{K}$ ) and let $f \in \mathcal{M}(\mathbb{K})$ (resp. $f \in \mathcal{M}(S)$, resp. $f \in \mathcal{M}(D))$. Then $T(r, f)=T(r, f(u x+c)$ ), $Z(r, f)=Z(r, f(u x+c)), N(r, f)=N(r, f(u x+c))$ whenever $r \geq|c|$ (resp. $T(r, f)=T(r, f(u x+c)), Z(r, f)=Z(r, f(u x+c)), N(r, f)=N(r, f(u x+c))$ whenever $r \geq|c|$, resp. $T_{R}(r, f)=T_{R}(r, f(u x+c)) Z_{R}(r, f)=Z_{R}(r, f(u x+c))$, $N_{R}(r, f)=N_{R}(r, f(u x+c))$ whenever $\left.r \geq|c|\right)$.

Notation: Let $f, g \in \mathcal{M}(\mathbb{K})$ (resp. let $f, g \in \mathcal{M}(S)$, resp. let $f, g \in$ $\mathcal{M}(D))$. We set $M(r, f)=\max ((Z(r, f), N(r, f))-N(r, f)(\operatorname{resp} . M(r, f)=$ $\max \left((Z(r, f), N(r, f))-N(r, f)\right.$, resp. $M_{R}(r, f)=\max \left(\left(Z_{R}(r, f), N_{R}(r, f)\right)-\right.$ $\left.N_{R}(r, f)\right)$.

Lemma 7 is also easily proven by the property: $M(r, f)=\max (\log (|f|(r)), 0)$, on $\mathcal{M}(\mathbb{K})$ and on $\mathcal{M}(S)$ and $M_{R}(r, f)=\max (\log (|f|(r)), 0)$ on $\mathcal{M}(D)$. 
Lemma 7: Let $f, g \in \mathcal{M}(\mathbb{K})$ (resp. let $f, g \in \mathcal{M}(S)$ ). Then $M(r, f g) \leq$ $M(r, f)+M(r, g), M(r, f+g) \leq M(r, f)+M(r, g), M\left(r, \frac{f^{\prime}}{f}\right) \leq O(1)$.

Let $f, g \in \mathcal{M}(D)$. Then $M_{R}(r, f g) \leq M_{R}(r, f)+M_{R}(r, g), M_{R}(r, f+g) \leq$ $M_{R}(r, f)+M_{R}(r, g), M_{R}\left(r, \frac{f^{\prime}}{f}\right) \leq O(1)$.

Lemmas 8 and 9 are easily proven:

Lemma 8: Let $f, g \in \mathcal{M}^{*}(\mathbb{K})$ (resp. let $f, g \in \mathcal{M}_{u}(S)$, resp. let $f, g \in$ $\left.\mathcal{M}^{*}(D)\right)$ have finitely many poles in $\mathbb{K}$ (resp. in $S$, resp. in $\left.D\right)$. Then $T(r, \lambda f+$ $\mu g) \leq \max (T(r, f), T(r, g))+O(1)($ resp. $T(r, \lambda f+\mu g) \leq \max (T(r, f), T(r, g))+$ $O(1)$, resp. $\left.T_{R}(r, \lambda f+\mu g) \leq \max \left(T_{R}(r, f), T_{R}(r, g)\right)+O(\log (r))\right)$.

Lemma 9: Let $f, g \in \mathcal{M}^{*}(\mathbb{K})$ (resp. let $f, g \in \mathcal{M}_{u}(S)$, resp. let $f, g \in$ $\mathcal{M}^{*}(D)$ ) have finitely many poles in $\mathbb{K}$ (resp. in $S$, resp. in D). Then $\left.Z\left(r, \frac{1}{f}-\frac{1}{g}\right) \leq Z(r, f-g)+O(\log (r))\right),\left(\right.$ resp. $Z\left(r, \frac{1}{f}-\frac{1}{g}\right) \leq Z(r, f-g)+O(1)$, resp. $\left.\left.Z_{R}\left(r, \frac{1}{f}-\frac{1}{g}\right) \leq Z_{R}(r, f-g)+O(\log (r))\right)\right)$.

\section{Proof of Theorems}

Proofs of Theorems 8.1 and 8.2: We will first prove Theorems 8.1 when $f$ and $g$ belong to $\mathcal{M}_{u}(S)$. So, let $f, g \in \mathcal{M}_{u}(S)$ sharing C.M. $q$ points $a_{j} \in$ $\mathbb{K}, 1 \leq j \leq q$. By Theorem 3.7, we have

$$
\left.(q-1) T(r, f) \leq \sum_{j=1}^{q} Z\left(r, f-a_{j}\right)+O(1) \leq Z(r, f-g)+O(1)\right)
$$

$$
\leq T(r, f)+T(r, g)+O(1)
$$

and similarly

$$
\begin{gathered}
(q-1) T(r, g) \leq \sum_{j=1}^{q} Z\left(r, g-a_{j}\right)+O(1) \leq Z(r, f-g)+O(1) \\
\leq T(r, f)+T(r, g)+O(1)
\end{gathered}
$$

hence

$$
(q-1)(T(r, f)+T(r, g)) \leq 2(T(r, f)+T(r, g))+O(1) .
$$

hence (3) implies $q \leq 3$.

Suppose now that $f$ and $g$ have finitely many poles. If $f$ and $g$ belong to $\mathcal{M}_{u}(S)$, by Lemma 8 , we have $Z(r, f-g) \leq \max (T(r, f), T(r, g))$, hence by $(1)$ and (2), now we obtain

$$
(q-1) \max (T(r, f), T(r, g)) \leq \max (T(r, f), T(r, g))+O(1)
$$

and hence $q \leq 2$. Consequently, since $q=3$, we have $f \equiv g$ in Theorem 8.2. 
If $f, g \in \mathcal{M}^{*}(D)$, the same reasoning holds.

Proof of Theorem 8.3: Let $f, g \in \mathcal{M}^{*}(\mathbb{K})$ be distinct and share three points $a_{j} \in \mathbb{K}, j=1,2,3$ C.M. Without loss of generality, we can assume that $a_{1} a_{2} \neq 0$. Suppose first that $f(x) \neq a_{3} \forall x \in \mathbb{K}$, hence $g(x) \neq a_{3} \forall x \in \mathbb{K}$. Let $F=\frac{1}{f}, G=\frac{1}{g}$ and let $b_{j}=\frac{1}{a_{j}} j=1,2$. Then $F$ and $G$ belong to to $\mathcal{A}(\mathbb{I K})$ and share $b_{1}$ and $b_{2}$ C.M and hence, by Theorem 5.1 we have $F \equiv G$, hence $f \equiv g$.

Thus, henceforth, we can assume that $f$ and $g$ take each value $a_{j}, j=1,2,3$ at least one time, therefore

$$
\left.Z\left(r, f-a_{j}\right) \geq \log (r)+O(1), r \in\right] 0,+\infty[, j=1,2,3 .
$$

Now we notice that

$$
\sum_{j=1}^{3} Z\left(r, f-a_{j}\right) \leq Z(r, f-g) \leq T(r, f)+T(r, g)
$$

Suppose first that $\lim _{r \rightarrow+\infty} \frac{Z\left(r, f-a_{j}\right)}{T(r, f)}=1 \forall j=1,2,3$. Then there exists $s>0$ such that $\frac{Z\left(r, f-a_{j}\right)}{T(r, f)} \geq \frac{3}{4} \forall j=1,2,3, \forall r>s$.

Then for every $r>s$, we have

$$
\frac{9}{4} T(r, f) \leq \sum_{j=1}^{3} Z\left(r, f-a_{j}\right) \leq Z(r, f-g)+O(1) \leq T(r, f)+T(r, g)+O(1)
$$

and similarly

$$
\frac{9}{4} T(r, g) \leq \sum_{j=1}^{3} Z\left(r, g-a_{j}\right) \leq Z(r, f-g)+O(1) \leq T(r, f)+T(r, g)+O(1) .
$$

Therefore, we obtain $\frac{9}{4}(T(r, f)+T(r, g)) \leq 2(T(r, f)+T(r, g))+O(1)$, a contradiction showing that $\liminf _{r \rightarrow+\infty} \frac{Z\left(r, f-a_{j}\right)}{T(r, f)}<1$ holds for at least one index.

Consequently, we are now led to assume there exists $\epsilon>0$ such that for a certain $k$, there exists a sequence of intervals $\left(\left[s_{n}, t_{n}\right]\right)_{n \in \mathbb{N}}$ such that $s_{n}<t_{n}<$ $s_{n+1}, \lim _{n \rightarrow+\infty} s_{n}=+\infty$ and $\frac{Z\left(r, f-a_{k}\right)}{T(r, f)} \leq 1-\epsilon \forall r \in\left[s_{n}, t_{n}\right], \forall n \in \mathbb{N}$. Without loss of generality, we can suppose $k=3$ and set $M=\bigcup_{n=1}^{+\infty}\left[s_{n}, t_{n}\right]$. So, when $r$ belongs to $M$, we have

$$
Z\left(r, f-a_{3}\right) \leq(1-\epsilon) T(r, f) \forall r \in M
$$

By Lemma 3 we can write $f$ in the form $\frac{\phi}{\psi}$ with $\phi, \psi \in \mathcal{A}(\mathbb{K})$ having no common zero. Then $N\left(r, f-a_{3}\right)=N(r, f)=Z(r, \psi)$, hence by (3), we have

$$
T\left(r, f-a_{3}\right)=T(r, f)=N(r, f) \forall r \in M .
$$


Now $Z\left(r, f-a_{j}\right)=Z\left(r, \phi-a_{j} \psi\right), \quad j=1,2,3, \quad N(r, f)=Z(r, \psi)$. Therefore by $(3)$, we have

$$
Z\left(r, \phi-a_{3} \psi\right) \leq(1-\epsilon) Z(r, \psi)
$$

By (4) and by Theorem 3.1, we can derive $\log \left(\left|\phi-a_{3} \psi\right|(r)\right) \leq(1-\epsilon) \log \left(\mid\left(a_{3}-\right.\right.$ $\left.\left.a_{1}\right) \psi \mid(r)\right)+O(1)$ and hence $\log \left(\left|\phi-a_{3} \psi\right|(r)\right)<\log \left(\left|\left(a_{3}-a_{1}\right) \psi\right|(r)\right)$ when $r$ is big enough in $M$. So, there exists $u \geq s$ such that $\log \left(\left|\phi-a_{3} \psi\right|(r)\right)<$ $\log \left(\left|\left(a_{3}-a_{1}\right) \psi\right|(r)\right) \forall r \in M, r \geq u$. Consequently, $\log \left(\left|\phi-a_{1} \psi\right|(r)\right)=\log \mid\left(a_{3}-\right.$ $\left.a_{1}\right) \psi \mid(r) \forall r \in J, r \geq u$ and hence $Z\left(r, f-a_{1}\right) \geq Z(r, \psi)+O(1) \forall r \in M, r \geq u$, therefore

$$
Z\left(r, f-a_{1}\right)=T(r, f)+O(1) \forall r \in M, r \geq u \text {. }
$$

Similarly, we have

$$
Z\left(r, f-a_{2}\right)=T(r, f)+O(1) \forall r \in M, r \geq u .
$$

Consequently, by (5) and (6), we obtain $Z\left(r, f-a_{1}\right)+Z\left(r, f-a_{2}\right) \geq 2 T(r, f)+$ $O(1) r \in J, r \geq u$ and hence $\sum_{j=1}^{3} Z\left(r, f-a_{j}\right) \geq 2 T(r, f)+Z\left(r, f-a_{3}\right)+$ $O(1) r \in M, r \geq u$, therefore by (2)

$$
2 T(r, f)+Z\left(r, f-a_{3}\right) \leq T(r, f)+T(r, g)+O(1) r \in M, r \geq u
$$

and similarly

$$
2 T(r, g)+Z\left(r, g-a_{3}\right) \leq T(r, f)+T(r, g)+O(1) r \in M, r \geq u \text {. }
$$

Thus,

$2(T(r, f)+T(r, g))+Z\left(r, f-a_{3}\right)+Z\left(r, g-a_{3}\right) \leq 2(T(r, f)+T(r, g))+O(1), r \in M, r \geq u$.

But then, by (1) we have a contradiction that finishes the proof.

Proof of Theorem 8.4: Without loss of generality, we can assume $a b \neq 0$. Let $F=\frac{1}{f}, G=\frac{1}{g}$ and let $a^{\prime}=\frac{1}{a}, b^{\prime}=\frac{1}{b}$. Then $F$ and $G$ share 0, $a, b$ C.M. Suppose that $F$ and $G$ are not identical. By Theorems 3.7 and 4.10 , we have $2 T(r, F) \leq Z(r, F)+Z\left(r, F-a^{\prime}\right)+Z\left(r, F-b^{\prime}\right)+O(1) \leq Z(r, F-G)+O(1)$ (resp. $2 T_{R}(r, F) \leq Z_{R}(r, F)+Z_{R}\left(r, F-a^{\prime}\right)+Z_{R}\left(r, F-b^{\prime}\right)+O(\log (r)) \leq$ $\left.Z_{R}(r, F-G)+O(\log (r))\right)$ hence by Lemma $9,2 T(r, F) \leq T(r, f-g)+O(1)$ (resp. $2 T_{R}(r, F) \leq T_{R}(f-g)$ ) and similarly, $2 T(r, G) \leq T(r, f-g)+O(1)$, (resp. $\left.2 T_{R}(r, G) \leq T_{R}(r, f-g)+O(\log (r))\right)$. Consequently, by Theorem 3.2 and 4.6 and Lemma 8, we have $2 \max (T(r, f), T(r, g) \leq \max (T(r, f), T(r, g)+O(1)$ (resp. $2 \max \left(T_{R}(r, f), T_{R}(r, g) \leq \max \left(T_{R}(r, f), T_{R}(r, g)+O(\log (r))\right)\right.$, a contradiction since $f, g$ belong to $\mathcal{M}_{u}(S)$, (resp. to $\mathcal{M}^{*}(D)$ ). Consequently, $F \equiv G$, and hence $f \equiv g$.

Proof of Theorem 8.5: Let $H \in \mathbb{K}(x)$ (resp. let $H \in \mathcal{M}_{b}(S)$, resp. $H \in$ $\left.\mathcal{M}^{z}(D)\right)$ be such that the function $V(x)=\frac{H(x) \prod_{j=1}^{k}\left(f(x)-\alpha_{j}\right)}{\prod_{j=1}^{k}\left(g(x)-\alpha_{j}\right)}$ have no pole 
and no zero in $\mathbb{K}$ (resp. in $S$, resp. in $D$ ). Then by Lemma $3, V$ is a constant in $\mathbb{I K}$ (resp. $V$ belongs to $\mathcal{M}_{b}(S)$, resp. $V$ belongs to $\mathcal{M}^{z}(D)$ ).

On the other hand, since $f$ and $g$ have finitely many poles, in all hypotheses, we note that $N(r, f)=O(\log (r)), N(r, g)=O(\log (r))$. Since $f$ and $g$ share $S_{2}$ I.M., we can derive that each zero of $\left(f(x)-\beta_{1}\right)\left(f(x)-\beta_{2}\right)$ in $\mathbb{K}$ (resp. in $S$, resp. in $D)$ is a zero of one of the following three functions:

$$
H^{-1} V-1, \quad H^{-1} V-\prod_{j=1}^{k} \frac{\beta_{1}-\alpha_{j}}{\beta_{2}-\alpha_{j}}, \quad H^{-1} V-\prod_{j=1}^{k} \frac{\beta_{2}-\alpha_{j}}{\beta_{1}-\alpha_{j}} .
$$

We can then derive that one of the following three cases $a), b), c$ ) must occur:

$$
\begin{gathered}
\text { a) } \prod_{j=1}^{k} \frac{f(x)-\alpha_{j}}{g(x)-\alpha_{j}} \equiv 1 \\
\text { b) } \prod_{j=1}^{k} \frac{f(x)-\alpha_{j}}{g(x)-\alpha_{j}} \equiv \prod_{j=1}^{k} \frac{\beta_{1}-\alpha_{j}}{\beta_{2}-\alpha_{j}} \\
\text { c) } \prod_{j=1}^{k} \frac{f(x)-\alpha_{j}}{g(x)-\alpha_{j}} \equiv \prod_{j=1}^{k} \frac{\beta_{2}-\alpha_{j}}{\beta_{1}-\alpha_{j}} .
\end{gathered}
$$

Indeed, suppose that none of the cases $a), b), c$ ) occurs. Then the following three inequalities are satisfied:

$$
\begin{gathered}
\text { (i) } \prod_{j=1}^{k}\left(\frac{f(x)-\alpha_{j}}{g(x)-\alpha_{j}}\right) \not \equiv 1 \\
\text { (ii) } \quad \prod_{j=1}^{k}\left(\frac{f(x)-\alpha_{j}}{g(x)-\alpha_{j}}\right) \not \equiv \prod_{j=1}^{k}\left(\frac{\beta_{1}-\alpha_{j}}{\beta_{2}-\alpha_{j}}\right) \\
\text { (iii) } \quad \prod_{j=1}^{k}\left(\frac{f(x)-\alpha_{j}}{g(x)-\alpha_{j}}\right) \not \equiv \prod_{j=1}^{k}\left(\frac{\beta_{2}-\alpha_{j}}{\beta_{1}-\alpha_{j}}\right) .
\end{gathered}
$$

Suppose first, for simplicity, that $f$ and $g$ belong to $\mathcal{M}^{*}(\mathbb{K})$. Then, by Theorem 3.7, we have:

$$
\begin{gathered}
T(r, f) \leq \bar{Z}\left(r, f-\beta_{1}\right)+\bar{Z}\left(r, f-\beta_{2}\right)+\bar{N}(r, f)+O(\log (r)) \\
\leq \bar{Z}\left(r, H^{-1} V-1\right)+\bar{Z}\left(r, H^{-1} V-\prod_{j=1}^{k}\left(\frac{\beta_{1}-\alpha_{j}}{\beta_{2}-\alpha_{j}}\right)\right)+\bar{Z}\left(r, H^{-1} V-\prod_{j=1}^{k}\left(\frac{\beta_{2}-\alpha_{j}}{\beta_{1}-\alpha_{j}}\right)\right)+O(\log (r)) \\
\leq 3 T\left(r, H^{-1} V\right)+O(\log (r)) \leq O(\log (r))
\end{gathered}
$$

Consequently, $T(r, f)=O(\log (r))$ i.e. $f \in \mathbb{K}(x)$, a contradiction. Similarly, if $f$ and $g$ belong to $\mathcal{M}_{u}(S)$ or to $\mathcal{M}^{*}(D)$, following the same calculation, we obtain $f \in \mathcal{M}_{b}(S), f \in \mathcal{M}^{z}(D)$, the same contradiction. Therefore one of the cases $a), b), c$ ) must occur. We will examine them successively. 
Suppose $a$ ) is satisfied. Since $f$ and $g$ share $S_{2}$ I.M., thanks to Hypothsis $(\mathcal{H})$, we can see that $f(x)=\beta_{1}$ if and only if $g(x)=\beta_{1}$ and $f(x)=\beta_{2}$ if and only if $g(x)=\beta_{2}$. Consequently, $f$ and $g$ share both $\beta_{1}$ and $\beta_{2}$ I.M. Then, by Lemma 1 and Relation $a$ ), this implies that $f$ and $g$ share $\beta_{1}$ C.M. and that they share $\beta_{2}$ C.M. Moreover, relation $a$ ) shows that each pole of $f$ is a pole of $f$ of same order. Then by Theorem 8.4 we have $f \equiv g$.

Suppose case $b$ ) is satisfied. Since $f$ and $g$ share $S_{2}$ I.M., thanks to Hypothsis $(\mathcal{H})$, we can check again that $f(x)=\beta_{1}$ if and only if $g(x)=\beta_{2}$ and similarly, $f(x)=\beta_{2}$ if and only if $g(x)=\beta_{1}$. Next, by Lemmas $2,4,5, f$ takes every value infinitely many times. Hence there exists $u \in \mathbb{K}$ (resp. $u \in S$, resp. $u \in D$ ) such that $f(u)=\beta_{1}$ and hence $g(u)=\beta_{2}$. But then, by relation $b$ ), we have

$$
\left(\prod_{j=1}^{k}\left(\beta_{1}-\alpha_{j}\right)\right)^{2}=\left(\prod_{j=1}^{k}\left(\beta_{2}-\alpha_{j}\right)\right)^{2}
$$

a contradiction.

Suppose finally that case $c$ ) occurs: this is exactly symmetric to case $b$ ) by permuting $\beta_{1}$ and $\beta 2$. Consequently, case $a$ ) must occur and therefore $f=g$. That finishes the proof of Theorem 8.5.

Proof of Theorem 8.6. Let $F(x)=\prod_{j=1}^{k}\left(f(x)-\alpha_{j}\right)$ and $G(x)=\prod_{j=1}^{k}\left(g(x)-\alpha_{j}\right)$, let $\gamma_{1}=\prod_{j=1}^{k}\left(\beta_{1}-\alpha_{j}\right)$ and $\gamma_{2}=\prod_{j=1}^{k}\left(\beta_{2}-\alpha_{j}\right)$. By hypotheses of Theorem 8.6, $f$ and $g$ share the set $\left\{\alpha_{1}, \ldots, \alpha_{k}\right\}$ I.M. and the set $\left\{\beta_{1}, \beta_{2}\right\}$ C.M. hence $F$ and $G$ share 0 I.M. and share the set $\left\{\gamma_{1}, \gamma_{2}\right\}$ C.M. Moreover, by hypothesis, we have $\gamma_{1} \gamma_{2} \neq 0$ and $\gamma_{1}^{2} \neq \gamma_{2}^{2}$. We notice that $\gamma_{1}+\gamma_{2} \neq 0$, hence when $F$ has a zero, since $G$ also has a zero, $F(x)+G(x)$ cannot be equal to $\gamma_{1}+\gamma_{2}$. Consequently,

$$
F(x)+G(x) \not \equiv \gamma_{1}+\gamma_{2} .
$$

We will show that $F$ and $G$ share 0 C.M. The proof is obviously trivial when $F \equiv G$ and the case when $F$ or $G$ is constant is excluded by the hypotheses $f \in \mathcal{M}^{*}(\mathbb{K})$ and $g \in \mathcal{M}^{*}(\mathbb{K})$ (resp. $f \in \mathcal{M}_{u}(S)$ and $g \in \mathcal{M}_{u}(S)$, resp. $f \in \mathcal{M}^{*}(D)$ and $\left.g \in \mathcal{M}^{*}(D)\right)$. Thus, in the following, we assume that neither $F$ nor $G$ are constant and that $F \not \equiv G$.

Since $f$ and $g$ have finitely many poles in $\mathbb{K}$ (resp. in $S$, resp. in $D$ ), and since $F$ and $G$ share $\left\{\gamma_{1}, \gamma_{2}\right\}$ C.M., it is easily seen that the function $\frac{\left(F(x)-\gamma_{1}\right)\left(F(x)-\gamma_{2}\right)}{\left(G(x)-\gamma_{1}\right)\left(G(x)-\gamma_{2}\right)}$ has finitely many zeros and poles which only come from the poles of $f$ and $g$. Consequently, there exists a function $E \in \mathbb{K}(x)$ such that the function $W(x)=\frac{E(x)\left(F(x)-\gamma_{1}\right)\left(F(x)-\gamma_{2}\right)}{\left(G(x)-\gamma_{1}\right)\left(G(x)-\gamma_{2}\right)}$ have no zero and no pole in $\mathbb{I K}$ (resp. in $S$, resp. in $D)$. Consequently, $W$ belongs to $\mathbb{K}(x)\left(\operatorname{resp}\right.$. to $\mathcal{M}_{b}(S)$, resp to $\left.\mathcal{M}^{z}(D)\right)$.

We must now introduce three auxiliary functions. Put $M(x)=(F(x)-$ $G(x))\left(F(x)+G(x)-\gamma_{1}-\gamma_{2}\right)$ and 
$\phi_{1}(x)=M(x)\left(\frac{\gamma_{1}\left(\gamma_{1}-\gamma_{2}\right) F^{\prime}(x)}{F(x)\left(F(x)-\gamma_{1}\right)\left(F(x)-\gamma_{2}\right)}-\frac{\gamma_{2}\left(\gamma_{2}-\gamma_{1}\right) G^{\prime}(x)}{G(x)\left(G(x)-\gamma_{1}\right)\left(G(x)-\gamma_{2}\right)}\right)$,
$\phi_{2}(x)=M(x)\left(\frac{\gamma_{2}\left(\gamma_{2}-\gamma_{1}\right) F^{\prime}(x)}{F(x)\left(F(x)-\gamma_{1}\right)\left(F(x)-\gamma_{2}\right)}-\frac{\gamma_{1}\left(\gamma_{1}-\gamma_{2}\right) G^{\prime}(x)}{G(x)\left(G(x)-\gamma_{1}\right)\left(G(x)-\gamma_{2}\right)}\right)$
$\phi_{3}(x)=M(x)\left(\frac{F^{\prime}(x)}{F(x)\left(F(x)-\gamma_{1}\right)\left(F(x)-\gamma_{2}\right)}-\frac{G^{\prime}(x)}{G(x)\left(G(x)-\gamma_{1}\right)\left(G(x)-\gamma_{2}\right)}\right)$.

We will first prove that $\phi_{1}$ and $\phi_{2}$ are not identically zero. Suppose for simplicity that $f$ and $g$ belong to $\mathcal{M}(\mathbb{I K})$.

Indeed, suppose that $\phi_{1} \equiv 0$. By (1) and due to the fact that $F \not \equiv G$, we have

$$
\frac{\gamma_{1}\left(\gamma_{1}-\gamma_{2}\right) F^{\prime}(x)}{F(x)\left(F(x)-\gamma_{1}\right)\left(F(x)-\gamma_{2}\right)} \equiv \frac{\gamma_{2}\left(\gamma_{2}-\gamma_{1}\right) G^{\prime}(x)}{G(x)\left(G(x)-\gamma_{1}\right)\left(G(x)-\gamma_{2}\right)} .
$$

Let us remark that, by Lemma $2, F$ takes every value infinitely many times because $F$ has finitely many poles. Let $u$ be a zero of $F-\gamma_{1}$ of multiplicity $j$. Since $F$ and $G$ share $\left\{\gamma_{1}, \gamma_{2}\right\}$ C.M., either $u$ is a zero of $G-\gamma_{1}$ of same multiplicity, or it is a zero of $G-\gamma_{2}$ of same multiplicity.

Suppose first that $u$ is a zero of $G-\gamma_{1}$. By developing at $u$, we have $F(x)-\gamma_{1}=a_{j}(x-u)^{j}+a_{j+1}(x-u)^{j+1} \varphi(x)$ and $G(x)-\gamma_{1}=b_{j}(x-u)^{j}+$ $b_{j+1}(x-u)^{j+1} \psi(x)$ with $\varphi, \psi \in \mathcal{M}(\mathbb{K})$, having no pole at $u$.

Then by computing we can check that the residue of $\frac{\gamma_{1}\left(\gamma_{1}-\gamma_{2}\right) F^{\prime}}{F(x)\left(F(x)-\gamma_{1}\right)\left(F(x)-\gamma_{2}\right.}$ at $u$ is $j$ because $\frac{\gamma_{1}\left(\gamma_{1}-\gamma_{2}\right)}{F(u)\left(F(u)-\gamma_{2}\right)}=1$ and this of $\frac{\gamma_{2}\left(\gamma_{2}-\gamma_{1}\right) G^{\prime}}{G(x)\left(G(x)-\gamma_{1}\right)\left(G(x)-\gamma_{2}\right)}$ is $-\frac{j \gamma_{2}}{\gamma_{1}}$ because $\frac{\gamma_{2}\left(\gamma_{2}-\gamma_{1}\right)}{G(u)\left(G(u)-\gamma_{2}\right)}=-\frac{\gamma_{2}}{\gamma_{1}}$. So, we have $\frac{\gamma_{2}}{\gamma_{1}}=-1$, hence $\gamma_{1}^{2}=\gamma_{2}^{2}$, a contradiction to the hypothesis. Therefore, $u$ must be a zero of $G-\gamma_{2}$ of multiplicity $j$. So, by developing $F$ and $G$ at $u$ we obtain again expressions of the form

$F(x)-\gamma_{1}=a_{j}(x-u)^{j}+a_{j+1}(x-u)^{j+1} \varphi(x)$ and $G(x)-\gamma_{2}=b_{j}(x-u)^{j}+$ $b_{j+1}(x-u)^{j+1} \psi(x)$ with $\varphi, \psi \in \mathcal{M}(\mathbb{K})$ having no pole at $u$, hence we can check that the residue at $u$ of $\frac{\gamma_{1}\left(\gamma_{1}-\gamma_{2}\right) F^{\prime}}{F(x)\left(F(x)-\gamma_{1}\right)\left(F(x)-\gamma_{2}\right)}$ is $-\frac{j \gamma_{1}}{\gamma_{2}}$ and this of $\frac{\gamma_{2}\left(\gamma_{2}-\gamma_{1}\right) G^{\prime}}{G(x)\left(G(x)-\gamma_{1}\right)\left(G(x)-\gamma_{2}\right)}$ is $-\frac{j \gamma_{2}}{\gamma_{1}}$, hence we obtain again $\gamma_{1}^{2}=\gamma_{2}^{2}$, the same contradiction. This finishes proving that $\phi_{1} \not \equiv 0$. And similarly, we can see that $\phi_{2} \not \equiv 0$.

We will now prove that $\phi_{3} \equiv 0$. Indeed, suppose that $\phi_{3} \not \equiv 0$. We will prove that the poles of $\phi_{3}$ only come from the poles of $F$ and of $G$. Indeed, let $a$ be a pole of $\phi_{3}$ and suppose that it is not a pole of $F$ and $G$. Then it is a zero $F$ and $G$ or a zero of $\left(F-\gamma_{1}\right)\left(F-\gamma_{2}\right)$ leading to a zero of $\left(G-\gamma_{1}\right)\left(G-\gamma_{2}\right)$.

Suppose first that $a$ is a zero of $F$. Then it is also a zero of $G$ and hence that makes a pole of order 1 or zero for

$$
\frac{F^{\prime}}{F\left(F-\gamma_{1}\right)\left(F-\gamma_{2}\right)}-\frac{G^{\prime}}{G\left(G-\gamma_{1}\right)\left(G-\gamma_{2}\right)} .
$$

But since $a$ is also a zero for $F-G$, it is not a pole for $\phi_{3}$. Similarly, let $b$ be a zero for $F-\gamma_{1}$. It is then a pole of order 1 or zero for

$$
\frac{F^{\prime}}{F\left(F-\gamma_{1}\right)\left(F-\gamma_{2}\right)}-\frac{G^{\prime}}{G\left(G-\gamma_{1}\right)\left(G-\gamma_{2}\right)} \text {. }
$$


But since $F$ and $G$ share $\left\{\gamma_{1}, \gamma_{2}\right\}$ C.M, we can check that the function

$$
(F-G)\left(F+G-\gamma_{1}-\gamma_{2}\right)
$$

has a zero at $b$, hence $\phi_{3}$ has no pole at $b$. Similarly, of course, if $c$ is a zero of $F-\gamma_{2}$, then $\phi_{3}$ has no pole at $c$.

This finishes proving that each pole of $\phi_{3}$ must be a pole of $f$ or $g$. Next, if $f$ and $g$ belongs to $\mathcal{M}(S)$ or to $\mathcal{M}(D)$ we can make the same reasoning. Thus, in each hypothesis, we have $N\left(r, \phi_{3}\right) \leq O(\log (r))$ (resp. $N\left(r, \Phi_{3}\right) \leq O(1)$, resp. $\left.N_{R}\left(r, \phi_{3}\right) \leq O(\log (r))\right)$.

Henceforth, we will gather the two first hypotheses: $f, g \in \mathcal{M}(\mathbb{K})$ and $f, g \in \mathcal{M}(S)$ and we will consider next the hypothesis $f, g \in \mathcal{M}(D)$.

Let us develop $\phi_{3}$. We have:

$$
\begin{gathered}
\phi_{3}=\left(\frac{\left(F-\gamma_{1}\right)\left(F-\gamma_{2}\right)-\left(G-\gamma_{1}\right)\left(G-\gamma_{2}\right)}{\left(F-\gamma_{1}\right)\left(F-\gamma_{2}\right)}\right) \frac{F^{\prime}}{F}+\left(\frac{\left(G-\gamma_{1}\right)\left(G-\gamma_{2}\right)-\left(F-\gamma_{1}\right)\left(F-\gamma_{2}\right)}{\left(G-\gamma_{1}\right)\left(G-\gamma_{2}\right)}\right) \frac{G^{\prime}}{G} \\
=\left(1-E W^{-1}\right) \frac{F^{\prime}}{F}+\left(1-E^{-1} W\right) \frac{G^{\prime}}{G} .
\end{gathered}
$$

Therefore, by Lemma 7, we have

$$
\begin{gathered}
M\left(r, \phi_{3}\right) \leq M\left(r, 1-E W^{-1}\right)+M\left(r, \frac{F^{\prime}}{F}\right)+M\left(r, 1-E^{-1} W\right)+M\left(r, \frac{G^{\prime}}{G}\right) \leq 2 T\left(r, E W^{-1}\right)+O(1) \\
\leq O(\log (r))
\end{gathered}
$$

Now since $N\left(r, \phi_{3}\right) \leq O(\log (r))$, we can conclude that $T\left(r, \phi_{3}\right) \leq O(\log (r))$ (resp. since $N_{R}\left(r, \phi_{3}\right) \leq O(\log (r))$, we can conclude that $\left.T_{R}\left(r, \phi_{3}\right) \leq O(\log (r))\right)$.

Now, let us develop $\phi_{1}$ : we have

$$
\begin{gathered}
\phi_{1}=\left(\frac{\gamma_{1}\left(\gamma_{1}-\gamma_{2}\right)\left(F-\gamma_{1}\right)\left(F-\gamma_{2}\right)-\left(G-\gamma_{1}\right)\left(G-\gamma_{2}\right)}{\left(F-\gamma_{1}\right)\left(F-\gamma_{2}\right)}\right) \frac{F^{\prime}}{F} \\
+\left(\frac{\gamma_{2}\left(\gamma_{2}-\gamma_{1}\right)\left(G-\gamma_{1}\right)\left(G-\gamma_{2}\right)-\left(F-\gamma_{1}\right)\left(F-\gamma_{2}\right)}{\left(G-\gamma_{1}\right)\left(G-\gamma_{2}\right)}\right) \frac{G^{\prime}}{G} \\
\left.=\gamma_{1}\left(\gamma_{1}-\gamma_{2}\right)\right)\left(1-E W^{-1}\right) \frac{F^{\prime}}{F}+\gamma_{1}\left(\gamma_{1}-\gamma_{2}\right)\left(1-E^{-1} W\right) \frac{G^{\prime}}{G} .
\end{gathered}
$$

Therefore, as for $\phi_{3}$ we have $T\left(r, \phi_{1}\right) \leq O(\log (r))$ and similarly, $T\left(r, \phi_{2}\right) \leq$ $O(\log (r))$.

We will estimate $\bar{Z}\left(r, F-\gamma_{1}\right)$. Let $u$ be a zero of $F-\gamma_{1}$ of multiplicity $j$ : either it is a zero of multiplicity $j$ of $G-\gamma_{1}$ or it is a zero of multiplicity $j$ of $G-\gamma_{2}$ because $F$ and $G$ share the set $\left\{\gamma_{1}, \gamma_{2}\right\}$ C.M.

Suppose first that $u$ is a zero of $G-\gamma_{1}$ of multiplicity $j$. As it is a zero of multiplicity $j$ of $F-\gamma_{1}$, it is a zero of multiplicity at least $j$ of $F+G+\gamma_{1}+\gamma_{2}$. On the othe hand, by developing $\frac{F^{\prime}}{\left.F\left(F-\gamma_{1}\right)(F)-\gamma_{2}\right)}-\frac{G^{\prime}}{F^{\prime}}-\frac{\left.\gamma_{1}\right)\left(G-\gamma_{2}\right)}{G^{\prime}}$ at the point $u$, we can check that $\frac{F^{\prime}}{\left.F\left(F-\gamma_{1}\right)(F)-\gamma_{2}\right)}-\frac{G^{\prime}}{G\left(G-\gamma_{1}\right)\left(G-\gamma_{2}\right)}$ has no pole at $u$ because $u$ is a pole of same order for $\frac{F^{\prime}}{\left.F\left(F-\gamma_{1}\right)(F)-\gamma_{2}\right)}$ and for $\frac{G^{\prime}}{G\left(G-\gamma_{1}\right)\left(G-\gamma_{2}\right)}$. Consequently, $\phi_{3}$ has a zero of order at least $j$ at $u$. 
Suppose now that $u$ is a zero of order $j$ of $G-\gamma_{1}$. Then $u$ is a zero of order at least $j$ for $F+G-\gamma_{1}-\gamma_{2}$. Consider now $\phi_{1}$ : we can check that $\frac{\gamma_{1}\left(\gamma_{1}-\gamma_{2}\right) F^{\prime}}{\left.F\left(F-\gamma_{1}\right)(F)-\gamma_{2}\right)}-\frac{\gamma_{2}\left(\gamma_{2}-\gamma_{1}\right) G^{\prime}}{G\left(G-\gamma_{1}\right)\left(G-\gamma_{2}\right)}$ has no pole at $u$ and hence, as previously, $u$ is a zero of order at least $j$ for $\phi_{1}$.

Consequently, if $f, g$ belongs to $\mathcal{M}^{*}(\mathbb{I K})$ or to $\mathcal{M}_{u}(S)$, we have

$$
\bar{Z}\left(r, F-\gamma_{1}\right) \leq Z\left(r, \phi_{1}\right)+Z\left(r, \phi_{3}\right) \leq O(\log (r))
$$

Similarly and symmetrically, we obtain

$$
\bar{Z}\left(r, F-\gamma_{2}\right) \leq Z\left(r, \phi_{2}\right)+Z\left(r, \phi_{3}\right) \leq O(\log (r))
$$

Consequently, by (2) and (3), and by the second Main Theorem, we have

$$
T(r, F) \leq \bar{Z}\left(r, F-\gamma_{1}\right)+\bar{Z}\left(r, F-\gamma_{2}\right)+\bar{N}(r, F)+O(\log (r)) \leq O(\log (r))
$$

and therefore $F$ belongs to $\mathbb{K}(x)$, a contradiction. Thus the hypothesis made above: $\phi_{3} \not \equiv 0$ is wrong.

And similarly, if $\mathcal{M}^{*}(D)$, by the same reasoning, we obtain $\bar{Z}_{R}\left(r, F-\gamma_{1}\right) \leq$ $Z_{R}\left(r, \phi_{1}\right)+Z_{R}\left(r, \phi_{3}\right) \leq O(\log (r))$, and $\bar{Z}_{R}\left(r, F-\gamma_{2}\right) \leq Z_{R}\left(r, \phi_{2}\right)+Z_{R}\left(r, \phi_{3}\right) \leq$ $O(\log (r))$, therefore

$T_{R}(r, F) \leq \bar{Z}_{R}\left(r, F-\gamma_{1}\right)+\bar{Z}_{R}\left(r, F-\gamma_{2}\right)+\bar{N}_{R}(r, F)+O(\log (r)) \leq O(\log (r))$

and hence $F$ belongs to $\mathcal{M}^{z}(D)$, a contradiction. Thus the hypothesis made above: $\phi_{3} \not \equiv 0$ is wrong again.

Consequently, now we have

$$
\frac{F^{\prime}(x)}{F(x)\left(F(x)-\gamma_{1}\right)\left(F(x)-\gamma_{2}\right)} \equiv \frac{G^{\prime}(x)}{G(x)\left(G(x)-\gamma_{1}\right)\left(G(x)-\gamma_{2}\right)} .
$$

Consider a zero $u$ of $F$ of order $j$ which by hypothesis is also a zero of $G$. The development at $u$ shows that $G$ also admits $u$ as a zero of order $j$ and therefore $F$ and $G$ share 0 C.M. But then by Theorem 8.5 , we have $F \equiv G$. Therefore, $f$ and $g$ now share the set $S_{1}$ C.M., and hence, by Theorem 8.5 , we can conclude $f=g$.

Proof of Theorems 8.7: Suppose that $f^{n}(x) f^{m}(a x+b)$ and $g^{n}(x) g^{m}(a x+b)$ share a rational function $Q \in \mathbb{K}(x)$ C.M. and that $f$ and $g$ are two distinct meromorphic functions with finitely many poles. Set $F(x)=f^{n}(x) f^{m}(a x+b)$ and $G(x)=g^{n}(x) g^{m}(a x+b)$. Thus $F-Q$ and $G-Q$ are two meromorphic functions with finitely many poles having the same zeros with respectively the same multiplicity. Therefore, by Lemma $3, \frac{F-Q}{G-Q}$ is a rational function $h \in$ $\mathbb{K K}(x), h \not \equiv 1$. Consequently,

$$
G(x)=h F(x)+(1-h) Q(x) .
$$

and of course

$$
F(x)=\frac{1}{h} G(x)+\frac{Q}{h}(h-1) .
$$


Applying Corollary 7.2.b, by (1) and (2) we have

$$
\begin{gathered}
T(r, F) \leq \bar{Z}\left(r, \frac{1}{h} G\right)+\bar{Z}\left(r, \frac{1}{h} G+(h-1) \frac{Q}{h}\right)+\bar{N}(r, F)+o(T(r, F)) \\
=\bar{Z}(r, G)+\bar{Z}(r, F)+o(T(r, F)) .
\end{gathered}
$$

Now, by Lemma 1 , we have $\bar{Z}(r, G) \leq 2 Z(r, g) \forall r \geq|c|$ and $\bar{Z}(r, F) \leq$ $2 Z(r, f) \forall r \geq|c|$, hence by Lemma 6 we obtain $T(r, F) \leq 2(T(r, g)+T(r, f)) \forall r \geq$ $|c|$. Next, by Theorems 3.2 and 4.6, we have $T(r, F)=(n+m) T(r, f)+O(\log (r))$, hence $(n+m) T(r, f) \leq 2(T(r, g)+T(r, f))+O(\log (r))$. Similarly, we have $(n+m) T(r, g) \leq 2(T(r, f)+T(r, g))+O(\log (r))$. Therefore $(n+m)(T(r, f)+$ $T(r, g)) \leq 4(T(r, f)+T(r, g))+o(T(r, F))+o(T(r, G))+O(\log (r))$. But by Theorems $3.2 o(T(r, F))$ is equivalent to $o(T(r, F)), o(T(r, G))$ is equivalent to $o(T(r, g))$, therefore $(n+m)(T(r, f)+T(r, g)) \leq 4(T(r, f)+T(r, g))+$ $o(T(r, f)))+o(T(r, g))$, which implies $n+m \leq 4$. Consequently, $F \equiv G$ since $n+m \geq 5$.

Now, suppose that $Q$ is a nonzero constant and that $f$ and $g$ are entire functions. Then $\frac{F-Q}{G-Q}$ is a constant $\lambda \neq 1$ and we have

$$
G(x)=\lambda F(x)+Q(1-\lambda) .
$$

and of course

$$
F(x)=\frac{1}{\lambda} G(x)+\frac{Q}{\lambda}(\lambda-1) .
$$

We can apply Theorem 3.7 on $\mathcal{M}(\mathbb{K})$ and obtain

$T(r, F) \leq \bar{Z}(r, G)+\bar{Z}(r, G+\lambda-1)-\log (r)+O(1)=\bar{Z}(r, G)+\bar{Z}(r, F)-\log (r)+O(1)$.

On the other hand, since $f, g$ belong to $\mathcal{A}^{*}(K)$, by Theorems 3.2, we now have $T(r, F)=T\left(r, f^{n}\right)+T\left(r, f^{m}(a x+b)\right)=(n+m) T(r, f)+O(1)$ and similarly, $T(r, G)=(n+m) T(r, g)+O(1)$. Consequently, $(n+m)(T r, f)+T(r, g)) \leq$ $4(T r, f)+T(r, g))-2 \log (r)+O(1)$, which implies $n+m<4$. Consequently, if $f$ and $g$ belong to $\mathcal{A}^{*}(\mathbb{K})$, if $Q$ is a nonzero constant and if $n+m \geq 4$, then $F \equiv G$.

So, we have proven that in each case, we have $F \equiv G$. Now, set $\phi(x)=\frac{f(x)}{g(x)}$ and $\psi(x)=\phi(a x+b)$. Then we have $\phi^{n}(x) \psi^{m}(x) \equiv 1$, hence $\frac{1}{\psi^{m}} \equiv \phi^{n}$ and hence

$$
T\left(r, \frac{1}{\psi^{m}}\right)=T\left(r, \phi^{n}\right) .
$$

But $T\left(r, \frac{1}{\psi}\right)=T(r, \psi)=T(r, \phi) \forall r \geq|c|$. On the other hand, by Theorems $3.2 T\left(r, \phi^{n}\right)=n T(r, \phi), T\left(r, \psi^{m}\right)=m T(r, \psi)$ hence by $(7)$, since $m \neq n$, we can see that $T(r, \phi)=0$. Therefore, $\phi$ is a constant $t$ and hence $f=t g$.

Now, $F-Q$ belongs to $\mathcal{M}^{*}(\mathbb{K})$, has finitely many poles and, by Lemma 2 , admits infinitely many zeros that are not zeros of $Q$. Let $c$ be such a zero. Then 
$c$ is also a zero of $G-Q$ and hence we have $f^{n}(c) f^{m}(a c+b)=g^{n}(c) g^{m}(a c+b)$, therefore $f^{n}(c) f^{m}(a c+b)=t^{n} f^{n}(c) t^{m} f^{m}(a c+b)$ i.e. $F(c)=t^{n+m} F(c)$. But since $c$ is not a zero of $Q$, we have $F(c) \neq 0$, hence $t^{n+m}=1$, which ends the proof.

Proof of Theorems 8.8: The proof of Theorem 8.8 roughly follows that of Theorem 8.7. Suppose first that $f^{n}(x) f^{m}(a x+b)$ and $g^{n}(x) g^{m}(a x+b)$ share C.M. a function $\theta \in \mathcal{M}(S)$ (resp. $\theta \in \mathcal{M}(D)$ ) having finitely many zeros and poles in $S$ (resp. in $D$ ), and are two distinct functions lying in $\mathcal{M}_{u}(S)$ (resp. in $\mathcal{M}^{*}(D)$ ) and have finitely many poles in $S$ (resp. in $D$ ). Set again $F(x)=f^{n}(x) f^{m}(a x+b)$ and $G(x)=g^{n}(x) g^{m}(a x+b)$. By Lemma $4, F-\theta$ and $G-\theta$ belong to $\mathcal{M}_{u}(S)$ (resp. to $\mathcal{M}^{*}(D)$ ) and are two functions having the same zeros with respectively the same multiplicity and have finitely many poles. Therefore $\frac{F-\theta}{G-\theta}$ belongs to $\mathcal{M}(S)$ (resp. to $\mathcal{M}(D)$ ) and has finitely many zeros and poles in $S$ (resp. in $D$ ). Consequently, it is a function $w$ that belongs to $\mathcal{M}_{b}(S)$ (resp. to $\mathcal{M}^{z}(D)$ ).

Suppose first $f, g \in \mathcal{M}(S)$. Then by Corollary 7.2.b, similarly to the proof of Theorem 8.7, we obtain

$$
T(r, F) \leq \bar{Z}(r, G)+\bar{Z}\left(r, G+\left(\frac{1}{w}-1\right) \theta+o(T(r, F))(r \rightarrow R)=\bar{Z}(r, G)\right.
$$

$$
+\bar{Z}(r, F)+o(T(r, F))(r \rightarrow R)
$$

Now, by Lemma 1 , we have again $\bar{Z}(r, G) \leq 2 Z(r, g)+O(1)$ and $\bar{Z}(r, F) \leq$ $2 Z(r, f)+O(1)$, hence by Lemma 6 we obtain $T(r, F) \leq 2(T(r, g)+T(r, f)+O(1)$ hence, by Theorems 3.2, $(n+m) T(r, g) \leq 2(T(r, g)+T(r, f))+O(1)$ and similarly $(n+m) T(r, f) \leq 2(T(r, f)+T(r, g))+O(1)$. On the other hand, $o(T(r, F))=o(T(r, f)), o(T(r, G))=o(T(r, g))$, therefore by (1), we obtain

$$
(n+m)(T r, f)+T(r, g)) \leq 4(T r, f)+T(r, g))+o(T(r, f))+o(T(r, g))
$$

and hence $n+m<5$, which is excluded by hypotheses. Consequently, we get $F \equiv G$. Putting again $\phi(x)=\frac{f(x)}{g(x)}$ and $\psi(x)=\phi(a x+b)$, we have $\frac{1}{\psi^{m}} \equiv \phi^{n}$, therefore in $S$ we get $T\left(r, \frac{1}{\psi^{m}}\right)=T\left(r, \phi^{n}\right)$ which implies that $T(r, \phi)=0$ since $n \neq m$. Thus $\phi$ is a constant $t$ again and hence, since $\theta$ has finitely many zeros in $S$, by Lemma 4 we can conclude as in Theorem 8.7 .

Similarly, if $f, g \in \mathcal{M}(D)$ we can make the same reasonning thanks to Lemma 5.

Proof of Theorem 8.9: Suppose first that $f$ and $w$ belong to $\mathcal{M}^{*}(\mathbb{I K})$ and set $h(x)=f(a x+b), F(x)=f(x)^{n} h^{m}(x)$. By Corollary 7.2.a we have

$$
T(r, F) \leq \bar{Z}(r, F)+\bar{Z}(r, F-w)+\bar{N}(r, F)+o(T(r, F))(r \rightarrow+\infty) .
$$

Clearly,

$$
\bar{Z}(r, F) \leq Z(r, f)+Z(r, h) \text {. }
$$


and

(3)

$\bar{N}(r, F) \leq N(r, f)+N(r, h)+N(r, w)=N(r, f)+N(r, h)+o(T(r, f))(r \rightarrow+\infty)$.

On the other hand, by Theorems 3.2, T(r, $\left.f^{n} h^{m}\right) \geq\left|T\left(r, f^{n}\right)-T\left(r, h^{m}\right)\right|_{\infty}$ and by Lemma 6 , for every $r \geq|b|$ we have $T(r, h)=T(r, f), Z(r, f)=$ $Z(r, h), N(r, f)=N(r, h)$, hence

$T(r, F) \geq|n-m|_{\infty} T(r, f)$. Moreover, $o(T(r, F))=o(T(r, f))$. Therefore, by $(1),(2)$ and (3) we obtain $\left(|n-m|_{\infty}-4\right) T(r, f) \leq \bar{Z}(r, F-w)+o(T(r, f))(r \rightarrow$ $+\infty)$ which proves the claim whenever $|n-m|_{\infty} \geq 5$.

Suppose now that $f$ and $w$ belong to $\mathcal{M}(S)$. Keeping the same notations, we can get the same relations $(1),(2),(3)$ and next $\left(|n-m|_{\infty}-4\right) T(r, f) \leq \bar{Z}(r, F-$ $w)+o(T(r, f))(r \rightarrow R)$, which proves again the claim whenver $|n-m|_{\infty} \geq 5$.

Finally, suppose that $f$ and $w$ belong to $\mathcal{M}(D)$ and keep the same notations. By Corollary 7.2.a now we have

$$
T_{R}(r, F) \leq \bar{Z}_{R}(r, F)+\bar{Z}_{R}(r, F-w)+\bar{N}_{R}(r, F)+o(T(r, F))
$$

Next, using Theorem 4.6 instead of 3.2 , we obtain here: $\left(|n-m|_{\infty}-\right.$ 4) $T_{R}(r, f) \leq \bar{Z}_{R}(r, F-w)+o\left(T_{R}(r, f)\right)(r \rightarrow+\infty)$. Thus, if $|n-m|_{\infty} \geq 5$ we have $T_{R}(r, f) \leq \bar{Z}_{R}(r, F-w)+o\left(T_{R}(r, f)\right)(r \rightarrow+\infty)$ which shows again that $F-w$ has infinitely many zeros.

Proof of Theorem 8.10: The beginning of the proof is similar to that of Theorem 8.9. Suppose first that $f \in \mathcal{M}^{*}(\mathbb{K})$ and set $h(x)=f(a x+b)$, $F(x)=f(x)^{n} h^{m}(x)$. Suppose that $F$ admits 4 distinct perfectly branched values $a_{1}, a_{2}, a_{3}, a_{4}$. By Theorem 3.7, we have

$$
3 T(r, F) \leq \sum_{j=1}^{4} \bar{Z}\left(r, F-a_{j}\right)+\bar{N}(r, F)-\log (r)+O(1)(r \rightarrow+\infty)
$$

hence

$3 T(r, F) \leq\left(\frac{1}{2}\right) \sum_{j=1}^{4} Z\left(r, F-a_{j}\right)+N(r, f)+N(r, h)-\log (r)+O(1)(r \rightarrow+\infty)$,

and hence

$$
\left.3\left(|n-m|_{\infty}\right) T(r, f) \leq\left(\frac{1}{2}\right) 4(n+m) T(r, f)\right)+2 T(r, f)+O(\log (r))(r \rightarrow+\infty),
$$

therefore $3|n-m|_{\infty} \leq 2(n+m)+2+\lambda(r)$, with $\lim _{r \rightarrow+\infty} \lambda(r)=0$. Consequently, if $3|n-m|_{\infty}>2(n+m+1)$, the function $F$ does not admit 4 perfectly branched values. Moreover, if $F$ admits 4 totally branched values $a_{1}, a_{2}, a_{3}, a_{4}$, then by (1) now we can get

$\left.\left.3\left(|n-m|_{\infty}\right) T(r, f) \leq\left(\frac{1}{2}\right) 4(n+m) T(r, f)\right)+2 T(r, f)-\log (r)\right)+O(1)(r \rightarrow+\infty)$, 
therefore $3|n-m|_{\infty}<2(n+m)+2$. Consequently, if $3|n-m|_{\infty} \geq 2(n+m+1)$, the function $F$ does not admit 4 totally branched values.

Suppose now that $F$ admits 3 distinct perfectly branched values $a_{1}, a_{2}, a_{3}$. By Theorem 3.7, we have

$$
2 T(r, F) \leq \sum_{j=1}^{3} \bar{Z}\left(r, F-a_{j}\right)+\bar{N}(r, F)-\log (r)+O(1)(r \rightarrow+\infty)
$$

and hence $\left.2\left(|n-m|_{\infty}\right) T(r, f) \leq\left(\frac{1}{2}\right) 3(n+m) T(r, f)\right)+2 T(r, f)+\lambda(r)$ with $\lim _{r \rightarrow+\infty} \lambda(r)=0,(r \rightarrow+\infty)$, therefore $2|n-m|_{\infty} \leq\left(\frac{3}{2}\right)(n+m)+2$. Consequently, if $4|n-m|_{\infty}>3(n+m)+4$, the function $F$ does not admit 3 perfectly branched values.

Moreover if $F$ admits 3 totally branched values $a_{1}, a_{2}, a_{3}$, then by (2) now we can get

$\left.2\left(|n-m|_{\infty}\right) T(r, f) \leq\left(\frac{1}{2}\right) 3(n+m) T(r, f)\right)+2 T(r, f)-\log (r)+O(1)(r \rightarrow+\infty)$,

therefore $4|n-m|_{\infty}<3(n+m)+4$. Consequently, if $4|n-m|_{\infty} \geq 3(n+m)+4$, the function $F$ does not admit 3 totally branched values.

Finally, if $f$ belongs to $\mathcal{M}_{u}(S)$ or to $\mathcal{M}^{*}(D)$, using in each case Theorem 3.7 or 4.10 , we can do the same reasoning for perfectly branched values. But Theorem 7.2 here does not show the term $-\log (r)+O(1)$ which is essential when we consider totally branched values. This is why we must replace $\geq$ by >in each statement for functions in $\mathcal{M}_{u}(S)$ or in $\mathcal{M}^{*}(D)$ concerning totally branched values.

Acknowledgements We are grateful to the referee for pointing out to us many misprints.

\section{References}

[1] T. T. H. An, Defect relation for non-Archimedean analytic maps into arbitrary projective varieties, Proc. Amer. Math. Soc. 135 (2007), 1255-1261.

[2] K. Boussaf, Motzkin factorization in algebras of analytic elements, Annales Mathématiques Blaise Pascal 2, n1, p.73-91. (1995).

[3] A. Boutabaa, Théorie de Nevanlinna p-adique, Manuscripta Math. 67, p. 251-269 (1990).

[4] A. Boutabaa and A. Escassut, URS and URSIMS for p-adic meromorphic functions inside a disk, Proc. of the Edinburgh Mathematical Society 44, p. 485-504 (2001).

[5] K. S. Charak, Value distribution theory of meromorphic functions, Mathematics Newsletter, Vol. 18, no. 4, pp. 1-35 (2009). 
[6] J. F. Chen, Uniqueness of meromorphic functions sharing two finite sets, Paper to appear.

[7] A. Escassut, Value Distribution in p-adic Analysis. WSCP Singapore, (2015).

[8] A. Escassut and T. T. H. An, p-adic Nevanlinna Theory outside of a hole, Vietnam Journal of Mathematics 45, n. 4, p. 681-694 (2017).

[9] H. K. Ha, On p-adic meromorphic functions, Duke Mathematical Journal, 50, 695-711 (1983).

[10] M. O. Hanyak and A. A. Kondratyuk, Meromorphic functions in m-punctured complex planes, Matematychni Studii 27, 1, p. 53-69 (2007).

[11] Vu Hoai An, Pham Ngoc Hoa and Ha Huy Khoai, Value sharing problems for differential polynomials of meromorphic functions ina nonArchimedean field, Paper to appear.

[12] M. Krasner, Prolongement analytique uniforme et multiforme dans les corps valués complets. Les tendances géométriques en algèbre et théorie des nombres, Clermont-Ferrand, p.94-141 (1964). Centre National de la Recherche Scientifique (1966), (Colloques internationaux de C.N.R.S. Paris, 143).

[13] Liu Gang and Meng Chao, Uniqueness for the Difference Monomials of P-Adic Entire Functions, Paper to appear.

[14] E. Motzkin, La décomposition d'un élément analytique en facteurs singuliers, Ann. Inst. Fourier 27, n 1, p. 67-82 (1977).

[15] X.G. Qi, L.Z. Yang and K. Liu, Uniqueness and periodicity of meromorphic functions concerning the difference operator, Comput. Math. Appl. 60, p. 1739-1746 (2010).

[16] K. Yamanoi, The second main theorem for small functions and related problems Acta Mathematica 192, p. 225-294 (2004).

\author{
Alain ESCASSUT \\ Université Clermont Auvergne, \\ Laboratoire de Mathématiques Blaise Pascal BP 10448, \\ CNRS, UMR 6620, LM, \\ F-63171 AUBIERE, \\ FRANCE \\ mail: alain.escassut@math.univ-bpclermont.fr \\ Ta Thi Hoai An \\ Associate Professor \\ Institute of Mathematics \\ Vietnam Academy of Science and Technology \\ 18, Hoang Quoc Viet \\ HANOI \\ VIETNAM \\ mail: tthan@math.ac.vn
}

